\title{
Sobolev versus Hölder local minimizers and existence of multiple solutions for a singular quasilinear equation
}

\section{JACQUES GIACOMONi, IAN SCHINDLER AND PETER TAKÁČ}

\begin{abstract}
We investigate the following quasilinear and singular problem,

$$
\begin{cases}-\Delta_{p} u=\frac{\lambda}{u^{\delta}}+u^{q} & \text { in } \Omega \\ \left.u\right|_{\partial \Omega}=0, \quad u>0 & \text { in } \Omega,\end{cases}
$$

where $\Omega$ is an open bounded domain with smooth boundary, $1<p<\infty, p-1<$ $q \leq p^{*}-1, \lambda>0$, and $0<\delta<1$. As usual, $p^{*}=\frac{N p}{N-p}$ if $1<p<$ $N, p^{*} \in(p, \infty)$ is arbitrarily large if $p=N$, and $p^{*}=\infty$ if $p>N$. We employ variational methods in order to show the existence of at least two distinct (positive) solutions of problem $(\mathrm{P})$ in $W_{0}^{1, p}(\Omega)$. While following an approach due to Ambrosetti-Brezis-Cerami, we need to prove two new results of separate interest: a strong comparison principle and a regularity result for solutions to problem (P) in $C^{1, \beta}(\bar{\Omega})$ with some $\beta \in(0,1)$. Furthermore, we show that $\delta<1$ is a reasonable sufficient (and likely optimal) condition to obtain solutions of problem $(\mathrm{P})$ in $C^{1}(\bar{\Omega})$.
\end{abstract}

Mathematics Subject Classification (2000): $35 \mathrm{~J} 65$ (primary); 35J20, 35J70 (secondary).

\section{Introduction}

In this paper we are interested in the following quasilinear and singular problem:

$$
\begin{cases}-\Delta_{p} u=\frac{\lambda}{u^{\delta}}+u^{q} & \text { in } \Omega \\ \left.u\right|_{\partial \Omega}=0, \quad u>0 & \text { in } \Omega\end{cases}
$$

Here, $\Omega$ is an open bounded domain with smooth boundary, $\Delta_{p} u=\nabla \cdot\left(|\nabla u|^{p-2} \nabla u\right)$ denotes the $p$-Laplace operator, $1<p<\infty, p-1<q \leq p^{*}-1, \lambda>0$, and $0<\delta<1$. As usual, $p^{*}=\frac{N p}{N-p}$ if $1<p<N, p^{*} \in(p, \infty)$ is arbitrarily large 
if $p=N$, and $p^{*}=\infty$ if $p>N$. Such problems arise, for instance, in models of pseudoplastic flows.

Our main concern is the question of existence and multiplicity of weak solutions to the Dirichlet boundary value problem $(\mathrm{P})$ in $W_{0}^{1, p}(\Omega)$. To obtain multiple (at least two distinct, positive) solutions of problem (P), we combine some wellknown variational methods (see e.g. Ambrosetti-Brezis-Cerami [2]) with a few new ideas of our own which employ two new results of separate interest: a regularity result for solutions to problem (P) in $C^{1, \beta}(\bar{\Omega})$ with some $\beta \in(0,1)$, Theorem 2.2 , and a strong comparison principle, Theorem 2.3. Our regularity result is obtained by adapting some ideas from Lieberman [33] for estimates in Campanato spaces. The strong comparison principle extends a result of Cuesta and Takáč [14]. More precisely, we look for solutions to problem (P) that are critical points of the energy functional $E_{\lambda}: W_{0}^{1, p}(\Omega) \rightarrow \mathbb{R}$ defined by

$$
\begin{aligned}
E_{\lambda}(u)= & \frac{1}{p} \int_{\Omega}|\nabla u|^{p} \mathrm{~d} x \\
& -\frac{\lambda}{1-\delta} \int_{\Omega}\left(u^{+}\right)^{1-\delta} \mathrm{d} x-\frac{1}{q+1} \int_{\Omega}\left(u^{+}\right)^{q+1} \mathrm{~d} x
\end{aligned}
$$

in the Sobolev space $W_{0}^{1, p}(\Omega)$. As usual, $r^{+}=\max \{r, 0\}$ and $r^{-}=\max \{-r, 0\}$ for $r \in \mathbb{R}$. Note that $E_{\lambda}$ is not of class $C^{1}$ on $W_{0}^{1, p}(\Omega)$ because of the singular term $\left(u^{+}\right)^{1-\delta}$; consequently, one cannot directly apply classical variational methods, such as the Mountain Pass lemma of Ambrosetti-Rabinowitz [4].

First, we show that the number

$$
\Lambda \stackrel{\text { def }}{=} \inf \{\lambda>0:(\mathrm{P}) \text { has no weak solution }\}
$$

satisfies $0<\Lambda<\infty$. Then we prove the existence of multiple (at least two distinct, positive) solutions of problem (P) for every $\lambda \in(0, \Lambda)$ : a local minimizer and a saddle point for the functional $E_{\lambda}$. Indeed, this existence and multiplicity result is a consequence of a competition between the positive and two negative terms in the energy functional $E_{\lambda}$. Notice that $E_{\lambda}(0)=0$ and $0<1-\delta<1<p<q+1$. Let $0<\lambda<\Lambda$. The first negative term,

$$
-\frac{\lambda}{1-\delta} \int_{\Omega}\left(u^{+}\right)^{1-\delta} \mathrm{d} x,
$$

dominates provided $u>0$ is "small", the positive term,

$$
\frac{1}{p} \int_{\Omega}|\nabla u|^{p} \mathrm{~d} x,
$$

becomes dominant for $u>0$ "mid-sized", and the second negative term,

$$
-\frac{1}{q+1} \int_{\Omega}\left(u^{+}\right)^{q+1} \mathrm{~d} x,
$$


becomes dominant for $u>0$ "large". This intuitive picture clearly suggests two critical points for $E_{\lambda}$ : a local minimizer between "small" and "mid-sized", and a saddle point between "mid-sized" and "large". As $\lambda \in(0, \Lambda)$ approaches $\Lambda$, the two critical points merge into a single one for $\lambda=\Lambda$ which disappears for $\lambda>\Lambda$; see definition (1.2).

The local minimizer is obtained first in the $C^{1}$ topology with the help of our $C^{1, \beta}$ regularity result (Theorem 2.2 ) combined with our strong comparison principle (Theorem 2.3). Then we take advantage of arguments due to Brezis and Nirenberg [12] and Ambrosetti, Brezis, and Cerami [2] in order to show that the local minimizer in the $C^{1}$ topology is also a local minimizer for $E_{\lambda}$ in the $W_{0}^{1, p}$ topology. In contrast, the saddle point is obtained by a modification of the Mountain Pass lemma of Ambrosetti-Rabinowitz [4], cf. Ghoussoub and Preiss [25].

Before giving our main results, let us briefly recall the literature concerning related singular problems. When $p=2$, the following problem has been investigated in quite a large number of papers:

$$
\left\{\begin{array}{rlrl}
-\Delta u & =\frac{\lambda \kappa(x)}{u^{\delta}}+\mu(x) u^{q} & & \text { in } \Omega ; \\
\left.u\right|_{\partial \Omega} & =0, \quad u>0 & \text { in } \Omega .
\end{array}\right.
$$

The weights $\kappa, \mu: \Omega \rightarrow \mathbb{R}$ are assumed to be nonnegative and (essentially) bounded. When $\mu=0$ (the purely singular problem), Crandall, Rabinowitz, and Tartar [15] show that, for any $\delta>0$, problem (1.3) admits a unique solution $u_{\lambda}$ in $C^{2}(\Omega) \cap$ $C(\bar{\Omega})$; furthermore, if $0<\delta<1$ then $u_{\lambda}$ is in $C^{2}(\Omega) \cap C^{1}(\bar{\Omega})$. When $\mu>0$ is small enough, Coclite and Palmieri [13] prove the existence of a solution to problem (1.3) for $0<\lambda<\Lambda$, with $\Lambda$ as in (1.2), $0<\Lambda<\infty$. Assuming $0<\delta<1$, Yijing, Shaoping, and Yiming [44] apply variational arguments based on Nehari's method [35] to show the existence of at least two solutions for $q>1$ subcritical: $q<\infty$ if $N=1$ or 2 , and $q<2^{*}-1=\frac{N+2}{N-2}$ if $N \geq 3$. The critical case $q=2^{*}-1$ and $N \geq 3$ was settled almost simultaneously in Haitao [29] and Hirano, Saccon, and Shioji [31] by two different methods: Perron's method and Nehari's method, respectively. To get the existence of at least two solutions, Haitao [29] shows that for any $0<\lambda<\Lambda$, the solution obtained by Perron's method is a local minimizer for the energy functional $E_{\lambda}$. His arguments depend on the strong maximum principle (see Brezis and Nirenberg [11, Theorem 3]). In Adimurthi and Giacomoni [1], the existence of at least two solutions in dimension $N=2$ is extended to $0<\delta<3$ and to critical nonlinearities of Trudinger-Moser type (see Moser [34]). Note that $\delta<3$ is the optimal condition on $\delta(\delta>0)$ to obtain solutions in $W_{0}^{1,2}(\Omega)$.

In the case $p \neq 2$ the question of multiplicity of solutions has been investigated for problems with convex and concave nonlinearities of the following kind:

$$
\left\{\begin{aligned}
-\Delta_{p} u & =\lambda u^{\delta}+u^{q} & & \text { in } \Omega \\
\left.u\right|_{\partial \Omega} & =0, \quad u>0 & & \text { in } \Omega,
\end{aligned}\right.
$$

where $0<\delta<p-1<q \leq p^{*}-1$. Ambrosetti, García Azorero, and Peral [3] establish the existence of at least two solutions to problem (1.4) for the subcritical 
$\left(q<p^{*}-1\right)$ and radially symmetric case $\left(\Omega=B_{R}(0)\right.$ a ball). Their main tools are some uniform a priori estimates (that require radial symmetry) and global bifurcation theory. The critical case $q=p^{*}-1$ is treated in García Azorero and Peral [20] with additional restrictions on $p$ and $\lambda>0$ small enough. These restrictions are used to prove that the levels of certain Palais-Smale sequences are strictly below the first critical level $S^{N / p} / N$ at which the Palais-Smale condition fails. Recall

$$
S=\inf _{0 \neq u \in W_{0}^{1, p}(\Omega)} \frac{\int_{\Omega}|\nabla u|^{p} \mathrm{~d} x}{\left(\int_{\Omega}|u|^{p^{*}} \mathrm{~d} x\right)^{p / p^{*}}} .
$$

Note that in this work, the existence of at least two solutions is not obtained for all $\lambda \in(0, \Lambda)$; only for $\lambda>0$ small enough. The restriction that $\lambda>0$ be small was removed in García Azorero, Peral, and Manfredi [21] using the approach of $C^{1}$ versus $W_{0}^{1, p}$ local minimizers ([2]). Essential elements in their approach are a $C^{1, \beta}$ regularity result of DiBenedetto [19] and a strong comparison principle of Guedda and Veron [27]. A similar result for problem (P) with radial symmetry is obtained in Giacomoni and Sreenadh [22] when $0<\delta, \lambda>0$ is small enough, and $q>p-1>0$. The radially symmetric setting enables a shooting method to be employed; see also Atkinson and Peletier [8] and Prashanth and Sreenadh [37] for $1<p<N$ and $\lambda \in(0, \Lambda)$.

The outline of this paper is as follows. Our main results are stated in Section 2. In Section 3 we prove the existence of a solution that is a local minimizer of $E_{\lambda}$ in $W_{0}^{1, p}(\Omega)$ for $0<\lambda<\Lambda$. In the proof we use Theorem 2.2 which follows from the regularity results contained in Appendices A and B. In Section 4, using Ekeland's principle and minimax arguments, we prove the existence of a second solution and thus finish the proof of Theorem 2.1.

ACKNOWLEDGEMENTS. The authors thank the anonymous referee for suggesting a number of valuable improvements and corrections.

\section{Main results}

We look for weak solutions (solutions, for short) of problem (P), that is, for functions $u \in W_{0}^{1, p}(\Omega)$ satisfying $\operatorname{ess~} \inf _{K} u>0$ over every compact set $K \subset \Omega$ and

$$
\int_{\Omega}|\nabla u|^{p-2} \nabla u \cdot \nabla \phi \mathrm{d} x=\lambda \int_{\Omega} u^{-\delta} \phi \mathrm{d} x+\int_{\Omega} u^{q} \phi \mathrm{d} x
$$

for all $\phi \in C_{\mathrm{c}}^{\infty}(\Omega)$. As usual, $C_{\mathrm{c}}^{\infty}(\Omega)$ denotes the space of all $C^{\infty}$ functions $\phi: \Omega \rightarrow \mathbb{R}$ with compact support. We denote by $p^{*}=N p /(N-p)$ the critical Sobolev exponent for $1<p<N$; we take $p^{*} \in(p, \infty)$ arbitrarily large for $p=N$, and $p^{*}=\infty$ for $p>N$. 
We introduce some notation which will be used throughout the paper. Given $1 \leq p<\infty$, the norm in $L^{p}(\Omega)$ is denoted by

$$
\|u\|_{L^{p}(\Omega)} \stackrel{\text { def }}{=}\left(\int_{\Omega}|u|^{p} \mathrm{~d} x\right)^{1 / p}
$$

and the norm in $W_{0}^{1, p}(\Omega)$ by

$$
\|u\|_{W_{0}^{1, p}(\Omega)} \stackrel{\text { def }}{=}\left(\int_{\Omega}|\nabla u|^{p} \mathrm{~d} x\right)^{1 / p} .
$$

The normalized positive eigenfunction associated with the principal eigenvalue $\lambda_{1}$ of $-\Delta_{p}$ is denoted by $\phi_{1}$ :

$$
-\Delta_{p} \phi_{1}=\lambda_{1}\left|\phi_{1}\right|^{p-2} \phi_{1} \quad \text { in } \Omega ; \quad \phi_{1}=0 \quad \text { on } \partial \Omega,
$$

$\phi_{1} \in W_{0}^{1, p}(\Omega)$ is normalized by $\phi_{1}>0$ in $\Omega$ and $\int_{\Omega} \phi_{1}^{p} \mathrm{~d} x=1$.

The function $d(x)$ denotes the distance from a point $x \in \bar{\Omega}$ to the boundary $\partial \Omega$, where $\bar{\Omega}=\Omega \cup \partial \Omega$ is the closure of $\Omega \subset \mathbb{R}^{N}$. This means that

$$
d(x) \stackrel{\text { def }}{=} \operatorname{dist}(x, \partial \Omega) \equiv \inf _{y \in \partial \Omega}|x-y| .
$$

Note that the strong maximum and boundary point principles from Vázquez [43, Theorem 5, page 200] guarantee $\phi_{1}>0$ in $\Omega$ and $\frac{\partial \phi_{1}}{\partial v}<0$ on $\partial \Omega$, respectively. Hence, since $\phi_{1} \in C^{1}(\bar{\Omega})$, there are constants $\ell$ and $L, 0<\ell<L$, such that $\ell d(x) \leq \phi_{1}(x) \leq L d(x)$ for all $x \in \Omega$.

The open ball in $W_{0}^{1, p}(\Omega)$ of radius $r$ centered at $u$ is denoted by

$$
\mathcal{B}_{r}(u) \stackrel{\text { def }}{=}\left\{v \in W_{0}^{1, p}(\Omega):\|u-v\|_{W_{0}^{1, p}(\Omega)}<r\right\}
$$

for some $r \in(0, \infty)$ and $u \in W_{0}^{1, p}(\Omega)$. If $u=0$, we abbreviate $\mathcal{B}_{r} \equiv \mathcal{B}_{r}(0)$. Finally, the open ball in $\mathbb{R}^{N}$ of radius $r$ centered at $x$ is denoted by $B_{r}(x)$.

Our main result is the following theorem.

Theorem 2.1. Let the pair $(p, q)$ satisfy either $p \in(1, \infty)$ and $q \in\left(p-1, p^{*}-1\right)$, or else $p \in\left(\frac{2 N}{N+2}, 2\right] \cup\left(\frac{3 N}{N+3}, 3\right)$ and $q=p^{*}-1$. Then there exists $\Lambda \in(0, \infty)$ with the following properties:

(i) For every $0<\lambda<\Lambda$ there exist at least two solutions of problem $(\mathrm{P}), u_{\lambda}$ and $v_{\lambda}$, such that $u_{\lambda}, v_{\lambda} \in C^{1}(\bar{\Omega})$ and $u_{\lambda} \supsetneqq v_{\lambda}$.

(ii) For $\lambda=\Lambda$ there exists at least one solution of $(\mathrm{P})$ in $C^{1}(\bar{\Omega})$.

(iii) For every $\lambda>\Lambda$ there is no solution of $(\mathrm{P})$. 
To prove Theorem 2.1, we establish a $C^{1, \beta}(\bar{\Omega})$, Theorem B.1 in Appendix B. Theorem B.1 gives the following regularity result for weak solutions to problem (P).

Theorem 2.2. Let $0<\delta<1,1<p<\infty$, and $p-1<q \leq p^{*}-1$. Then any weak solution to problem $(\mathrm{P})$ belongs to $C^{1, \beta}(\bar{\Omega})$ for some $\beta \in(0,1)$.

This regularity result motivates and complements the following new strong comparison principle.

Theorem 2.3. Let $u, v \in C^{1, \beta}(\bar{\Omega})$, for some $0<\beta<1$, satisfy $0 \supsetneqq u, 0 \supsetneqq v$ and

$$
\begin{aligned}
& -\Delta_{p} u-\lambda u^{-\delta}=f, \\
& -\Delta_{p} v-\lambda v^{-\delta}=g,
\end{aligned}
$$

with $u=v=0$ on $\partial \Omega$, where $f, g \in C(\Omega)$ are such that $0 \leq f<g$ pointwise everywhere in $\Omega$. Then, the following strong comparison principle holds:

$$
0<u<v \text { in } \Omega \text { and } \frac{\partial v}{\partial v}<\frac{\partial u}{\partial v}<0 \text { on } \partial \Omega
$$

Remark 2.4. Theorem 2.3 holds if we replace the $p$-Laplacian operator by a more general quasilinear operator; see, for instance, conditions (3)-(7) in Cuesta and Takáč [14].

Proof of Theorem 2.3. First, note that from the strong maximum of Vázquez (see Theorem 5 in [43]), we infer that $u>0$ in $\Omega$ and $\frac{\partial u}{\partial v}<0$ on $\partial \Omega$. Hence, since $u \in C^{1}(\bar{\Omega})$, there are constants $\ell$ and $L, 0<\ell<L$, such that $\ell d(x) \leq u(x) \leq$ $L d(x)$ near the boundary $\partial \Omega$. Analogous results hold for $v$. Moreover, $f \leq g$ in $\Omega$ guarantees $u \leq v$ in $\Omega$, by the weak comparison principle which can be proved by a standard variational argument. Consequently,

$$
\ell d(x) \leq u(x) \leq v(x) \leq L d(x)
$$

near the boundary $\partial \Omega$. As in the proof of Proposition 2.4 in Cuesta and Takáč [14] (see page 729), we define an $\eta$-neighborhood $\Omega_{\eta} \subset \Omega$ of the boundary $\partial \Omega$,

$$
\Omega_{\eta} \stackrel{\text { def }}{=}\{x \in \Omega: d(x)<\eta\},
$$

for $\eta>0$, and set $w \stackrel{\text { def }}{=} v-u, 0 \leq w \in C^{1, \beta}(\bar{\Omega})$ with $w=0$ on $\partial \Omega$. There exists $\eta>0$ small enough, such that in the open set $\Omega_{\eta}$ we have

$$
\begin{aligned}
& -\operatorname{div}(A(x) \nabla w)-B(x) w \\
& =-\sum_{i, j=1}^{N} \frac{\partial}{\partial x_{i}}\left(a_{i j}(x) \frac{\partial w}{\partial x_{j}}\right)-\lambda B(x) w=g-f>0 .
\end{aligned}
$$


The coefficients $a_{i j}(x)$ are given by

$$
\begin{aligned}
a_{i j}(x)= & \int_{0}^{1}|(1-t) \nabla u(x)+t \nabla v(x)|^{p-2} \\
& \times\left[\delta_{i j}+(p-2) \frac{\frac{\partial}{\partial x_{i}}((1-t) u+t v) \frac{\partial}{\partial x_{j}}((1-t) u+t v)}{|(1-t) \nabla u(x)+t \nabla v(x)|^{2}}\right] \mathrm{d} t
\end{aligned}
$$

for $x \in \Omega_{\eta}$ and $i, j=1,2, \ldots, N$, where $\delta_{i j}$ denotes the Kronecker symbol: $\delta_{i j}=1$ if $i=j ; \delta_{i j}=0$ if $i \neq j$. The differential operator above induced by the matrix $\left(a_{i j}\right)_{i, j=1,2, \ldots, N}$ is uniformly elliptic in $\Omega_{\eta}$ with $a_{i j} \in C^{0, \beta}\left(\overline{\Omega_{\eta}}\right)$ provided $\eta>0$ is chosen small enough. The coefficient $B(x)$ satisfies

$$
B(x)=-\delta \int_{0}^{1} \frac{\mathrm{d} t}{((1-t) u(x)+t v(x))^{\delta+1}}<0 .
$$

Inequalities in (2.6) guarantee that $B(x)$ satisfies the conditions of Lemma 2.7 in Hernández, Mancebo, and Vega [30]. We conclude that the (classical) strong maximum principle applies to inequality (2.8) in each connected component of the open set $\Omega_{\eta}$, thus yielding inequalities (2.5) in $\Omega_{\eta}$.

Finally, we will show that $u<v$ throughout $\Omega$. Let $\eta^{\prime} \in(0, \eta)$ and $\tilde{\Omega} \stackrel{\text { def }}{=}$ $\Omega \backslash \overline{\Omega_{\eta^{\prime}}}$. Employing $w>0$ in $\Omega_{\eta}$, we can find $c>0$ such that $w \geq c$ on $\partial \tilde{\Omega} \subset \Omega_{\eta}$. Moreover, recalling $f, g \in C(\Omega)$ with $0 \leq f<g$ pointwise everywhere in $\Omega$, we can choose $c>0$ small enough, such that also

$$
\frac{\lambda}{u^{\delta}}-\frac{\lambda}{(u+c)^{\delta}} \leq g-f \quad \text { holds in } \tilde{\Omega} .
$$

It follows that $u+c \leq v$ on $\partial \tilde{\Omega}$ together with

$$
-\Delta_{p}(u+c)-\frac{\lambda}{(u+c)^{\delta}} \leq f+(g-f)=g=-\Delta_{p} v-\frac{\lambda}{v^{\delta}} \quad \text { in } \tilde{\Omega} .
$$

Consequently, we may apply the weak comparison principle (see Proposition 2.3 in [14]) in order to conclude that $u+c \leq v$ holds throughout $\tilde{\Omega}$. As $\Omega=\Omega_{\eta} \cup \tilde{\Omega}$, we have verified $u<v$ throughout $\Omega$.

\section{Existence of weak solutions}

\subsection{Existence of a solution for $0<\lambda \leq \Lambda$}

First, let us consider the following purely singular Dirichlet problem:

$$
\left\{\begin{aligned}
-\Delta_{p} u & =\lambda u^{-\delta} & & \text { in } \Omega \\
\left.u\right|_{\partial \Omega} & =0, \quad u>0 & & \text { in } \Omega
\end{aligned}\right.
$$


Recall $0<\delta<1$. By requiring " $u>0$ in $\Omega$ " we actually mean $\operatorname{essinf}_{K} u>0$ for any compact set $K \subset \Omega$. We look for a solution $u \in W_{0}^{1, p}(\Omega)$ that satisfies equation (3.1) in the sense of distributions. More precisely, if $u_{0} \in W_{0}^{1, p}(\Omega)$ is a distributional solution of problem (3.1), with ess $\inf _{K} u_{0}>0$ for any compact set $K \subset \Omega$, then $u_{0} \in C^{1}(\Omega)$ by interior regularity due (independently) to DiBenedetto [19, Theorem 2, page 829] and Tolksdorf [42, Theorem 1, page 127].

Lemma 3.1. Assume $0<\delta<1$ and $\lambda>0$. Then problem (3.1) has a unique weak solution in $W_{0}^{1, p}(\Omega)$ in the sense of distributions. This solution, denoted by $\underline{u}_{\lambda}$, satisfies $\underline{u}_{\lambda} \geq \epsilon_{\lambda} \phi_{1}$ a.e. in $\Omega$, where $\epsilon_{\lambda}>0$ is a constant.

Proof. First, we observe that an energy functional on $W_{0}^{1, p}(\Omega)$ formally corresponding to problem (3.1) can be given by

$$
\tilde{E}_{\lambda}(u) \stackrel{\text { def }}{=} \frac{1}{p} \int_{\Omega}|\nabla u|^{p} \mathrm{~d} x-\frac{\lambda}{1-\delta} \int_{\Omega}\left(u^{+}\right)^{1-\delta} \mathrm{d} x, \quad u \in W_{0}^{1, p}(\Omega) .
$$

Owing to the Poincaré inequality and $0<1-\delta<1<p<\infty$, this functional is coercive and weakly lower semicontinuous on $W_{0}^{1, p}(\Omega)$. It follows that $\tilde{E}_{\lambda}$ possesses a global minimizer $u_{0} \in W_{0}^{1, p}(\Omega)$. We have $u_{0} \not \equiv 0$ in $\Omega$, owing to $\tilde{E}_{\lambda}(0)=0>\tilde{E}_{\lambda}\left(\epsilon \phi_{1}\right)$ for every $\epsilon>0$ small enough.

Second, the polar decomposition $u=u^{+}-u^{-}$of any function $u \in W_{0}^{1, p}(\Omega)$ gives $\nabla u=\nabla u^{+}-\nabla u^{-}$. Thus, if $u_{0}$ is a global minimizer for $\tilde{E}_{\lambda}$, then so is its absolute value $\left|u_{0}\right|$, by $\tilde{E}_{\lambda}\left(\left|u_{0}\right|\right) \leq \tilde{E}_{\lambda}\left(u_{0}\right)$. The equality $\tilde{E}_{\lambda}\left(\left|u_{0}\right|\right)=\tilde{E}_{\lambda}\left(u_{0}\right)$ holds if and only if $u_{0}^{-}=0$ a.e. in $\Omega$, that is, if and only if $u_{0} \geq 0$ a.e. in $\Omega$. Thus, any global minimizer $u_{0}$ for $\tilde{E}_{\lambda}$ must satisfy $u_{0} \geq 0$ a.e. in $\Omega$. Equivalently, $u \in W_{0}^{1, p}(\Omega)_{+}$where

$$
W_{0}^{1, p}(\Omega)_{+} \stackrel{\text { def }}{=}\left\{u \in W_{0}^{1, p}(\Omega): u \geq 0 \text { a.e. in } \Omega\right\}
$$

stands for the positive cone in $W_{0}^{1, p}(\Omega)$.

Third, we will show that even $u_{0} \geq \epsilon \phi_{1}$ holds almost everywhere in $\Omega$ with a constant $\epsilon>0$ small enough. To this end, let us first remark that the Gâteaux derivative $\tilde{E}_{\lambda}^{\prime}\left(\epsilon \phi_{1}\right)$ of $\tilde{E}_{\lambda}$ at $\epsilon \phi_{1}$ exists and satisfies

$$
\begin{aligned}
\tilde{E}_{\lambda}^{\prime}\left(\epsilon \phi_{1}\right) & =-\Delta_{p}\left(\epsilon \phi_{1}\right)-\lambda\left(\epsilon \phi_{1}\right)^{-\delta}=\lambda_{1}\left(\epsilon \phi_{1}\right)^{p-1}-\lambda\left(\epsilon \phi_{1}\right)^{-\delta} \\
& =\left(\epsilon \phi_{1}\right)^{-\delta}\left(\lambda_{1}\left(\epsilon \phi_{1}\right)^{p-1+\delta}-\lambda\right) \\
& \leq-\frac{\lambda}{2}\left(\epsilon \phi_{1}\right)^{-\delta}<0
\end{aligned}
$$

whenever $\epsilon>0$ is small enough, say, $0<\epsilon \leq \epsilon_{\lambda}$. 
On the contrary to our claim above, suppose that the (nonnegative) function $v=\left(u_{0}-\epsilon_{\lambda} \phi_{1}\right)^{-}=\left(\epsilon_{\lambda} \phi_{1}-u_{0}\right)^{+}$does not vanish identically in $\Omega$. Denote

$$
\Omega^{+}=\{x \in \Omega: v(x)>0\} .
$$

Let us investigate the function $\xi(t) \stackrel{\text { def }}{=} \tilde{E}_{\lambda}\left(u_{0}+t v\right)$ of $t \in \mathbb{R}_{+}=[0, \infty)$. This function is convex thanks to the fact that the restriction of the functional $\tilde{E}_{\lambda}$ to the positive cone $W_{0}^{1, p}(\Omega)_{+}$is convex. We have $\xi(t) \geq \xi(0)$ for all $t \geq 0$. Furthermore, owing to $u_{0}+t v \geq \max \left\{u_{0}, t \epsilon_{\lambda} \phi_{1}\right\} \geq t \epsilon_{\lambda} \phi_{1}$ for $t>0$, the Gâteaux derivative $\tilde{E}_{\lambda}^{\prime}\left(u_{0}+t v\right)$ of $\tilde{E}_{\lambda}$ at $u_{0}+t v$ exists and yields $\xi^{\prime}(t)=\left\langle\tilde{E}_{\lambda}^{\prime}\left(u_{0}+t v\right), v\right\rangle$ for $t>0$. This derivative is nonnegative and nondecreasing. Consequently, for $0<t<1$ we have

$$
\begin{aligned}
0 & \leq \xi^{\prime}(1)-\xi^{\prime}(t)=\left\langle\tilde{E}_{\lambda}^{\prime}\left(u_{0}+v\right)-\tilde{E}_{\lambda}^{\prime}\left(u_{0}+t v\right), v\right\rangle \\
& =\int_{\Omega^{+}} \tilde{E}_{\lambda}^{\prime}\left(\epsilon_{\lambda} \phi_{1}\right) v \mathrm{~d} x-\xi^{\prime}(t) \\
& \leq-\frac{\lambda}{2} \int_{\Omega^{+}}\left(\epsilon_{\lambda} \phi_{1}\right)^{-\delta} v \mathrm{~d} x<0,
\end{aligned}
$$

by inequality (3.2) and $\xi^{\prime}(t) \geq 0$, a contradiction. We have verified $v \equiv 0$ in $\Omega$, that is, $u_{0} \geq \epsilon_{\lambda} \phi_{1}$ a.e. in $\Omega$.

Finally, we have proved that every global minimizer $u_{0}$ for $\tilde{E}_{\lambda}$ on $W_{0}^{1, p}(\Omega)$ must satisfy $u_{0} \geq \epsilon_{\lambda} \phi_{1}$ a.e. in $\Omega$. The functional $\tilde{E}_{\lambda}$ being strictly convex on $W_{0}^{1, p}(\Omega)_{+}$, we conclude that $u_{0}$ is the only critical point of $\tilde{E}_{\lambda}$ in $W_{0}^{1, p}(\Omega)_{+}$with the property ess $\inf _{K} u_{0}>0$ for any compact set $K \subset \Omega$. Consequently, $\underline{u}_{\lambda}=u_{0}$ provides the unique weak solution to problem (3.1).

Remark 3.2. In our proof of Lemma 3.1 above, $v_{0}=0$ is a critical point for the functional $v \mapsto \tilde{E}_{\lambda}\left(u_{0}+v\right)$ defined for all $v \in C_{\mathrm{c}}^{\infty}(\Omega)$ only. We have proved that the functional $\tilde{E}_{\lambda}$ restricted to $W_{0}^{1, p}(\Omega)_{+}$has precisely one critical point that stays away from zero, uniformly on any compact set $K \subset \Omega$, namely, the global minimizer $u_{0}$.

We obtain the following result regarding $\Lambda$.

Lemma 3.3. Let $0<\delta<1$ and $p-1<q \leq p^{*}-1$. Then $0<\Lambda<\infty$.

Proof. We give the proof only in the critical case, i.e. $q=p^{*}-1$. In the subcritical case, i.e., $q<p^{*}-1$, the proof is simpler since the energy functional $\underline{E}_{\lambda}$ defined below is weakly lower semicontinuous in $W_{0}^{1, p}(\Omega)$. Let $\underline{u}_{\lambda}$ be the unique solution to (3.1). Define

$$
f_{\lambda}(x, s) \stackrel{\text { def }}{=} \begin{cases}\lambda s^{-\delta}+s^{q} & \text { if } s>\underline{u}_{\lambda}(x) \\ \lambda\left(\underline{u}_{\lambda}(x)\right)^{-\delta}+\left(\underline{u}_{\lambda}(x)\right)^{q} & \text { if } s \leq \underline{u}_{\lambda}(x)\end{cases}
$$


Let $F_{\lambda}(x, s)=\int_{0}^{s} f_{\lambda}(x, t) \mathrm{d} t$. Define $\underline{E}_{\lambda}: W_{0}^{1, p}(\Omega) \rightarrow \mathbb{R}$ by

$$
\underline{E}_{\lambda}(u) \stackrel{\text { def }}{=} \frac{1}{p} \int_{\Omega}|\nabla u|^{p} \mathrm{~d} x-\int_{\Omega} F_{\lambda}(x, u) \mathrm{d} x .
$$

From Lemma A.4, $\underline{E}_{\lambda}$ is $C^{1}\left(W_{0}^{1, p}(\Omega), \mathbb{R}\right)$. We consider the following minimization problem:

$$
I_{\lambda}=\min _{u \in \overline{\mathcal{B}}_{r}} \underline{E}_{\lambda}(u)
$$

Clearly, we have $I_{\lambda}>-\infty$. Note that $\int_{\Omega}\left(\frac{1}{p}|\nabla u|^{p}-\frac{1}{q+1}|u|^{q+1}\right) \mathrm{d} x>0$ for every $u \in \partial \mathcal{B}_{r}$ provided $r>0$ is small enough. Fix such $r>0$; the other negative term in $\underline{E}_{\lambda}(u)$ may be made arbitrarily small by taking $\lambda>0$ small enough. Therefore we find $r$ and $\lambda$ such that

$$
\min _{u \in \partial \mathcal{B}_{r}} \underline{E}_{\lambda}(u)>0 .
$$

Moreover, since $\underline{E}_{\lambda}(t u)<0$ for $t$ small, we have

$$
I_{\lambda}<0 \text {. }
$$

Let $\left\{u_{n}\right\}_{n=1}^{\infty}$ be a minimizing sequence, i.e. $u_{n} \subset \mathcal{B}_{r}$ and $\underline{E}_{\lambda}\left(u_{n}\right) \rightarrow I_{\lambda}$ as $n \rightarrow$ $\infty$. From (3.7) and (3.8), $\left\{u_{n}\right\}_{n=1}^{\infty}$ satisfies $\operatorname{dist}\left(u_{n}, \partial \mathcal{B}_{r}\right) \geq \eta_{0}$ for some $\eta_{0}>0$. Therefore, there exists $0<r_{0}<r$ such that

$$
u_{n} \in \mathcal{B}_{r_{0}} .
$$

Now, from Ekeland's variational principle, there exist $r_{0} \leq r_{1}<r$ and a sequence $\left\{v_{n}\right\}_{n=1}^{\infty} \subset \mathcal{B}_{r_{1}}$ satisfying

$$
\begin{aligned}
& \operatorname{dist}\left(u_{n}, v_{n}\right) \leq \frac{1}{n}, \quad \underline{E}_{\lambda}\left(u_{n}\right) \leq \underline{E}_{\lambda}\left(v_{n}\right) \text { and } \\
& \underline{E}_{\lambda}^{\prime}\left(v_{n}\right) \rightarrow 0 \text { in } W^{-1, p^{\prime}}(\Omega) \text { as } n \rightarrow \infty .
\end{aligned}
$$

From the first statement of (3.10), $\left\{v_{n}\right\}_{n=1}^{\infty}$ is a minimizing sequence for $I_{\lambda}$ and up to a subsequence satisfies $v_{n} \rightarrow \tilde{u}_{\lambda}$ as $n \rightarrow \infty$ with $\tilde{u}_{\lambda} \in \overline{\mathcal{B}_{r_{1}}}$. From the last statement of (3.10), we have

$$
-\Delta_{p}\left(v_{n}\right)-f_{\lambda}\left(x, v_{n}\right)=o_{n}(1) \text { in } W^{-1, p^{\prime}}(\Omega) .
$$

From (3.11), Theorem 2.1 in Boccardo and Murat [9] with

$$
\begin{aligned}
& f_{n}(x)=\left(\max \left\{v_{n}(x), \underline{u}_{\lambda}(x)\right\}\right)^{-\delta}+o_{n}(1), \\
& g_{n}(x)=\left(\max \left\{v_{n}(x), \underline{u}_{\lambda}(x)\right\}\right)^{q}
\end{aligned}
$$


(note that from Hardy's inequality and since $q=p^{*}-1,\left\{f_{n}\right\}_{n=1}^{\infty}$ and $\left\{g_{n}\right\}_{n=1}^{\infty}$ satisfy the conditions in Theorem 2.1 in [9]), Remark 2.1 in [9] and from Brezis and Lieb [10], it follows that

$$
\begin{aligned}
\left\|v_{n}\right\|_{W_{0}^{1, p}(\Omega)} & =\left\|v_{n}-\tilde{u}_{\lambda}\right\|_{W_{0}^{1, p}(\Omega)}+\left\|\tilde{u}_{\lambda}\right\|_{W_{0}^{1, p}(\Omega)}+o_{n}(1) \text { and } \\
\left\|v_{n}\right\|_{L^{q+1}(\Omega)} & =\left\|v_{n}-\tilde{u}_{\lambda}\right\|_{L^{q+1}(\Omega)}+\left\|\tilde{u}_{\lambda}\right\|_{L^{q+1}(\Omega)}+o_{n}(1)
\end{aligned}
$$

as $n \rightarrow \infty$. From (3.9), (3.10) and (3.12), it follows that $\tilde{u}_{\lambda}, v_{n}-\tilde{u}_{\lambda} \in \mathcal{B}_{r}$. Thus,

$$
\int_{\Omega}\left(\frac{1}{p}\left|\nabla v_{n}-\tilde{u}_{\lambda}\right|^{p}-\frac{1}{q+1}\left|v_{n}-\tilde{u}_{\lambda}\right|^{q+1}\right) \mathrm{d} x>0 .
$$

From (3.12) and (3.13), we get

$$
\begin{aligned}
I_{\lambda} & =\underline{E}_{\lambda}\left(v_{n}\right)+o_{n}(1) \\
& =\underline{E}_{\lambda}\left(\tilde{u}_{\lambda}\right)+\frac{1}{p}\left\|v_{n}-\tilde{u}_{\lambda}\right\|_{W_{0}^{1, p}(\Omega)}^{p}-\frac{1}{q+1}\left\|v_{n}-\tilde{u}_{\lambda}\right\|_{L^{q+1}(\Omega)}^{q+1}+o_{n}(1) \\
& \geq \underline{E}_{\lambda}\left(\tilde{u}_{\lambda}\right)+o_{n}(1) .
\end{aligned}
$$

Hence, $\underline{E}_{\lambda}\left(\tilde{u}_{\lambda}\right)=I_{\lambda}$ and

$$
\left\{\begin{aligned}
-\Delta_{p} \tilde{u}_{\lambda} & =f_{\lambda}\left(x, \tilde{u}_{\lambda}\right) \text { in } \Omega ; \\
\left.\tilde{u}_{\lambda}\right|_{\partial \Omega} & =0 .
\end{aligned}\right.
$$

Now, Theorem 2.3 imply that $\tilde{u}_{\lambda}>\underline{u}_{\lambda}$ in $\Omega$, hence $\tilde{u}_{\lambda}$ is a weak solution to problem (P). Thus $\Lambda>0$.

Now, let us show that $\Lambda<\infty$. We argue by contradiction: suppose there exists a sequence $\lambda_{n} \rightarrow \infty$ such that problem (P) admits a solution $u_{n}$. There exists $\lambda_{*}>0$ such that

$$
\frac{\lambda}{t^{\delta}}+t^{q} \geq\left(\lambda_{1}+\epsilon\right) t^{p-1} \text { for all } t>0, \epsilon \in(0,1) \text { and } \lambda>\lambda_{*} .
$$

Choose $\lambda_{n}>\lambda_{*}$. Clearly $u_{n}$ is a supersolution of the problem

$$
\left\{\begin{array}{l}
-\Delta_{p} u=\left(\lambda_{1}+\epsilon\right) u^{p-1} \text { in } \Omega \\
u>0,\left.\quad u\right|_{\partial \Omega}=0 .
\end{array}\right.
$$

for all $\epsilon \in(0,1)$. We now use Lemma 3.1 to choose $\mu<\lambda_{1}+\epsilon$ small enough so that $\mu \phi_{1}(x)<u_{n}(x)$ and $\mu \phi_{1}$ is a subsolution to problem (3.14). By a monotone interation procedure we obtain a solution to (3.14) for any $\epsilon \in(0,1)$, contradicting the fact that $\lambda_{1}$ is an isolated point in the spectrum of $-\Delta_{p}$ in $W_{0}^{1, p}(\Omega)$ (see Anane [5]). 
We prove now the existence of positive weak solution to (P) for any $0<\lambda<$ $\Lambda$. Precisely, we have the following result:

Proposition 3.4. For any $\lambda \in(0, \Lambda)$, there exists $u_{\lambda}$ a positive weak solution to (P). Moreover, $E_{\lambda}\left(u_{\lambda}\right)<0$.

Proof. Fix $0<\lambda<\lambda_{2}<\Lambda$. $\lambda_{2}$ is such that there exist solutions to (P) for $\lambda=\lambda_{2}$. Let $\underline{u}_{\lambda}$ be the solution of (3.1) and $u_{\lambda_{2}}$ is one solution of (P) (when $\lambda=\lambda_{2}$ in the equation of (P)). Clearly, from Theorem B.1 in Appendix B, $\underline{u}_{\lambda}, u_{\lambda_{2}}$ are in $C^{1, \beta}(\bar{\Omega})$ for some $0<\beta<1$ and $\underline{u}_{\lambda} \leq u_{\lambda_{2}}$ in $\Omega$. Indeed, setting $\underline{\Omega} \stackrel{\text { def }}{=}\left\{\underline{u}_{\lambda}-\bar{u}>0\right\}$ and from the equations satisfied by $\underline{u}_{\lambda}$ and $\bar{u}=u_{\lambda_{2}}$ we have

$$
\int_{\underline{\Omega}}\left(+\Delta_{p} \bar{u}-\Delta_{p} \underline{u}_{\lambda}\right)\left(\underline{u}_{\lambda}-\bar{u}\right) \mathrm{d} x \leq \lambda \int_{\underline{\Omega}}\left(\underline{u}_{\lambda}^{-\delta}-\bar{u}^{-\delta}\right)\left(\underline{u}_{\lambda}-\bar{u}\right) \mathrm{d} x \leq 0
$$

and

$$
\begin{aligned}
& \int_{\underline{\Omega}}\left(+\Delta_{p} \bar{u}-\Delta_{p} \underline{u}_{\lambda}\right)\left(\underline{u}_{\lambda}-\bar{u}\right) \mathrm{d} x \\
& \geq \int_{\underline{\Omega}}\left(\left|\nabla \underline{u}_{\lambda}\right|^{p-2} \nabla \underline{u}_{\lambda}-|\nabla \bar{u}|^{p-2} \nabla \bar{u}\right)\left(\nabla \underline{u}_{\lambda}-\nabla \bar{u}\right) \mathrm{d} x \\
& \geq\left\{\begin{array}{l}
C_{p} \int_{\underline{u}_{\lambda}-\bar{u}>0} \frac{\left|\nabla\left(\underline{u}_{\lambda}-\bar{u}\right)\right|^{2}}{\left(\left|\nabla \underline{u}_{\lambda}\right|+|\nabla \bar{u}|\right)^{2-p}} \mathrm{~d} x \text { if } 1<p<2 \\
C_{p} \int_{\underline{u}_{\lambda}-\bar{u}>0}\left|\nabla\left(\underline{u}_{\lambda}-\bar{u}\right)\right|^{p} \mathrm{~d} x \text { if } p \geq 2
\end{array}\right. \\
& \geq 0
\end{aligned}
$$

from Lemma 4.1 in Ghoussoub and Yuan [26]. Hence from (3.15) and (3.16), we get $\underline{u}_{\lambda} \leq \bar{u}$.

By the strong comparison principle (Theorem 2.3), we obtain $\bar{u}>\underline{u}_{\lambda}$ in $\Omega, \frac{\partial \bar{u}}{\partial v}<\frac{\partial \underline{u} \lambda}{\partial v}$ on $\partial \Omega$. Define

$$
\tilde{f}_{\lambda}(x, s)= \begin{cases}\lambda \bar{u}(x)^{-\delta}+\bar{u}(x)^{q} & \text { if } s>\bar{u}(x), \\ \lambda s^{-\delta}+s^{q} & \text { if } \underline{u}_{\lambda}(x) \leq s \leq \bar{u}(x), \\ \lambda \underline{u}_{\lambda}(x)^{-\delta}+\underline{u}_{\lambda}(x)^{q} & \text { if } s<\underline{u}_{\lambda}(x) .\end{cases}
$$

Let $\tilde{F}_{\lambda}(x, s)=\int_{0}^{s} \tilde{f}_{\lambda}(x, t) \mathrm{d} t$. Define the functional $\tilde{E}_{\lambda}: W_{0}^{1, p}(\Omega) \rightarrow \mathbb{R}$ by

$$
\tilde{E}_{\lambda}(u)=\frac{1}{p} \int_{\Omega}|\nabla u|^{p} \mathrm{~d} x-\int_{\Omega} \tilde{F}_{\lambda}(x, u) \mathrm{d} x .
$$

$\tilde{E}_{\lambda}$ is bounded below in $W_{0}^{1, p}(\Omega)$ and is weakly lower semi-continuous. Hence, $\tilde{E}_{\lambda}$ achieves its global minimum at some $u_{\lambda} \in W_{0}^{1, p}(\Omega)$. Moreover, since $\tilde{E}_{\lambda}$ is 
$C^{1}$ by Lemma A.4, $u_{\lambda}$ solves the equation $-\Delta_{p} u_{\lambda}=\tilde{f}_{\lambda}\left(x, u_{\lambda}\right)$ in $\Omega$. From the strong maximum principle of Vázquez (see Theorem 5 in [43]) we get $u_{\lambda}>0$ in $\Omega$. It follows by regularity results (see again Theorem B.1 in Appendix B) that $u_{\lambda} \in C^{1, \beta}(\bar{\Omega})$ for some $\beta \in(0,1)$. Again, by Therorem 2.3, we conclude that $\underline{u}_{\lambda}<u_{\lambda}<\bar{u}$ in $\Omega$ and $\frac{\partial}{\partial v}\left(u_{\lambda}-\underline{u}_{\lambda}\right)<0, \frac{\partial}{\partial v}\left(\bar{u}-u_{\lambda}\right)<0$ on $\partial \Omega$. Hence, $\tilde{f}_{\lambda}\left(x, u_{\lambda}\right)=\lambda u_{\lambda}^{-\delta}+u_{\lambda}^{q}$ for $x \in \Omega$ and so $u_{\lambda}$ is a weak solution to (P). Moreover, we have that

$$
\tilde{E}_{\lambda}\left(u_{\lambda}\right) \leq \tilde{E}_{\lambda}\left(\underline{u}_{\lambda}\right)=E_{\lambda}\left(\underline{u}_{\lambda}\right)<\frac{1}{p} \int_{\Omega}\left|\nabla \underline{u}_{\lambda}\right|^{p} \mathrm{~d} x-\frac{\lambda}{1-\delta} \int_{\Omega} \underline{u}_{\lambda}^{1-\delta} \mathrm{d} x<0 .
$$

This completes the proof of Proposition 3.4.

Now, we show the following result.

Proposition 3.5. There exists at least one positive weak solution for $\lambda=\Lambda$ to $(\mathrm{P})$.

Proof. Let $\left\{\lambda_{k}\right\}_{k=1}^{\infty}$ such that $\lambda_{k} \nearrow \Lambda$ as $k \rightarrow \infty$. Then, from Proposition 3.4, there exists $u_{k}=u_{\lambda_{k}} \geq \underline{u}_{\lambda_{k}}$ to a weak positive solution to (P) for $\lambda=\lambda_{k}$. Therefore, for any $\phi \in C_{c}^{\infty}(\Omega)$, we have

$$
\int_{\Omega}\left|\nabla u_{k}\right|^{p-2} \nabla u_{k} \nabla \phi \mathrm{d} x=\lambda_{k} \int_{\Omega}\left(u_{k}\right)^{-\delta} \phi \mathrm{d} x+\int_{\Omega} u_{k}^{q} \phi \mathrm{d} x .
$$

Since $u_{k} \in W_{0}^{1, p}(\Omega)$ and $u_{k} \geq \underline{u}_{\lambda_{k}}$ it is easy to see that (3.17) holds also for $\phi \in W_{0}^{1, p}(\Omega)$. Moreover, from Proposition 3.4

$$
E_{\lambda_{k}}\left(u_{k}\right)<0 .
$$

From (3.18), it follows that

$$
\sup _{k}\left\|u_{k}\right\|_{W_{0}^{1, p}(\Omega)}<\infty .
$$

Hence, there exists $u_{\Lambda} \geq \underline{u}_{\lambda_{k}}$ such that $u_{k} \rightarrow u_{\Lambda}$ in $W_{0}^{1, p}(\Omega)$ as $k \rightarrow \infty$ and then by Sobolev imbedding:

$$
u_{k} \rightarrow u_{\Lambda} \text { in } L^{q}(\Omega) \text { and pointwise a.e. as } k \rightarrow \infty \text {. }
$$

From (3.17), (3.19) and (3.20), we get for any $\phi \in W_{0}^{1, p}(\Omega)$ :

$$
\int_{\Omega}\left|\nabla u_{\Lambda}\right|^{p-2} \nabla u_{\Lambda} \nabla \phi \mathrm{d} x=\lambda \int_{\Omega}\left(u_{\Lambda}\right)^{-\delta} \phi \mathrm{d} x+\int_{\Omega} u_{\Lambda}^{q} \phi \mathrm{d} x
$$

which completes the proof of Proposition 3.5. 
From the above propositions, we get the following corollary:

Corollary 3.6. Let $1<p<\infty, p-1<q \leq p^{*}-1,0<\delta<1$, and $0<\lambda \leq \Lambda$. Then there exists a minimal solution to $(\mathrm{P})$.

Proof. We use here the weak comparison principle (see Proposition 2.3 in Cuesta and Takáč [14] or Tolksdorf [41]) and the following monotone iterative scheme:

$$
\left\{\begin{array}{l}
-\Delta_{p} u_{n}-\frac{\lambda}{u_{n}^{\delta}}=u_{n-1}^{q} \text { in } \Omega ; \\
\left.u_{n}\right|_{\partial \Omega}=0,
\end{array}\right.
$$

where $u_{0}=\underline{u}_{\lambda}$, the unique solution to (3.1). Note that $u_{0}$ is a weak subsolution to $(\mathrm{P})$ and $u_{0} \leq u_{\Lambda}$ where $u_{\Lambda}$ is the solution to (P) obtained in Proposition 3.5. Then, from the weak comparison principle, we get easily that $u_{0} \leq u_{1}$ and $\left\{u_{n}\right\}_{n=1}^{\infty}$ is nondecreasing. Furthermore, $u_{n} \leq u_{\Lambda}$ and $\left\{u_{n}\right\}_{n=1}^{\infty}$ is uniformly bounded in $W_{0}^{1, p}(\Omega)$. Hence, it is easy to prove that $\left\{u_{n}\right\}_{n=1}^{\infty}$ converges weakly in $W_{0}^{1, p}(\Omega)$ and pointwise to $\hat{u}_{\lambda}$, a weak solution to $(\mathrm{P})$. Let us show that $\hat{u}_{\lambda}$ is the minimal solution to (P) for any $\lambda \in(0, \Lambda]$. Let $v_{\lambda}$ a weak solution to $(\mathrm{P})$ for $\lambda \in(0, \Lambda]$. Then, $u_{0}=\underline{u}_{\lambda} \leq v_{\lambda}$. From the weak comparison principle, $u_{n} \leq v_{\lambda}$ for any $n \geq 0$. Letting $n \rightarrow \infty$, we get $\hat{u}_{\lambda} \leq v_{\lambda}$. This completes the proof of Corollary 3.6.

\section{2. $C^{1}$ versus $W^{1, p}$ local minimizers of the energy}

Let $u_{\lambda}$ be the solution to (P) given by Proposition 3.4. The main result in this paragraph is

Proposition 3.7. For $0<\lambda<\Lambda$, $u_{\lambda}$ is a local minimizer of $\underline{E}_{\lambda}$ in $W_{0}^{1, p}(\Omega)$.

Proof. We observe first that $u_{\lambda}$ is a local minimizer in the $C^{1}$-topology. Indeed, taking advantage of the strong comparison principle shown in Theorem 2.3 and the definition of $u_{\lambda}$, we have that for $v>0$ small enough,

$$
\left\|u-u_{\lambda}\right\|_{C^{1}(\bar{\Omega})} \leq v \Rightarrow \underline{u}_{\lambda} \leq u \leq \bar{u} .
$$

where $\bar{u}$ is defined in the proof of Proposition 3.4. From (3.23), we get that

$$
\left\|u-u_{\lambda}\right\|_{C^{1}(\bar{\Omega})} \leq v \Rightarrow \underline{E}_{\lambda}\left(u_{\lambda}\right)=\tilde{E}_{\lambda}\left(u_{\lambda}\right) \leq \tilde{E}_{\lambda}(u)=\underline{E}_{\lambda}(u) .
$$

Now, let us show that $u_{\lambda}$ is a local minimizer of $\underline{E}_{\lambda}$ in $W_{0}^{1, p}(\Omega)$. Suppose not and we derive a contradiction. First, we deal with the subcritical case, i.e. $q<$ $p^{*}-1$. In this case, $\underline{E}_{\lambda}$ is weakly lower semicontinuous on $W_{0}^{1, p}(\Omega)$ and achieves its minimum on bounded subsets of $W_{0}^{1, p}(\Omega)$. Hence, if $u_{\lambda}$ is not a local minimum for $\underline{E}_{\lambda}$, for every $\epsilon>0$ we obtain $v_{\epsilon}$ such that $0<\left\|v_{\epsilon}\right\|_{W_{0}^{1, p}(\Omega)} \leq \epsilon$ and

$$
\underline{E}_{\lambda}\left(u_{\lambda}+v_{\epsilon}\right)<\underline{E}_{\lambda}\left(u_{\lambda}\right), \underline{E}_{\lambda}\left(u_{\lambda}+v_{\epsilon}\right)=\inf _{\|v\|_{W_{0}^{1, p}(\Omega)} \leq \epsilon} \underline{E}_{\lambda}\left(u_{\lambda}+v\right) .
$$


By the Lagrange multiplier rule (see Phelps [36]), we obtain $\mu_{\epsilon} \leq 0$ such that

$$
\left\langle\underline{E}_{\lambda}^{\prime}\left(u_{\lambda}+v_{\epsilon}\right), h\right\rangle=\mu_{\epsilon} \int_{\Omega}\left|\nabla v_{\epsilon}\right|^{p-2} \nabla v_{\epsilon} . \nabla h \mathrm{~d} x, \quad \forall h \in W_{0}^{1, p}(\Omega) .
$$

That is, in the weak sense,

$$
-\Delta_{p}\left(u_{\lambda}+v_{\epsilon}\right)-f_{\lambda}\left(x, u_{\lambda}+v_{\epsilon}\right)=-\mu_{\epsilon} \Delta_{p} v_{\epsilon}
$$

where $f_{\lambda}$ is defined in (3.4). Define the maps $A_{\epsilon}: \bar{\Omega} \times \mathbb{R}^{N} \rightarrow \mathbb{R}^{N}$ and $\tilde{h}_{\lambda}$ : $\bar{\Omega} \times \mathbb{R}^{N} \rightarrow \mathbb{R}$ as

$$
\begin{aligned}
A_{\epsilon}(x, w)= & \left|\nabla u_{\lambda}(x)+w\right|^{p-2}\left(\nabla u_{\lambda}(x)+w\right)-\left|\nabla u_{\lambda}(x)\right|^{p-2} \nabla u_{\lambda}(x) \\
& -\mu_{\epsilon}|w|^{p-2} w \text { with } \\
\tilde{h}_{\lambda}(x, s) \stackrel{\text { def }}{=} & f_{\lambda}\left(x, u_{\lambda}(x)+s\right)-f_{\lambda}\left(x, u_{\lambda}(x)\right) \\
= & \lambda\left(\max \left\{u_{\lambda}(x)+s, \underline{u}_{\lambda}(x)\right\}\right)^{-\delta}-\lambda u_{\lambda}(x)^{-\delta} \\
& +\left(\max \left\{u_{\lambda}(x)+s, \underline{u}_{\lambda}(x)\right\}\right)^{q}-u_{\lambda}(x)^{q} .
\end{aligned}
$$

Then (3.25) can be written as

$$
\left\{\begin{array}{c}
-\nabla \cdot\left(A_{\epsilon}\left(x, \nabla v_{\epsilon}\right)\right)=\tilde{h}_{\lambda}\left(x, v_{\epsilon}\right) \text { in } \Omega ; \\
v_{\epsilon}=0 \text { on } \partial \Omega .
\end{array}\right.
$$

Using similar arguments as in García Azorero, Peral, and Manfredi [21] (Section 2, page 5 in the subcritical case and pages 19-20 in the critical case), the fact that the singular terms in (3.26) are non increasing and arguments in the proof of Theorem A1 in García Azorero and Peral [20], we obtain that $\sup _{\epsilon}\left\|v_{\epsilon}\right\|_{L^{\infty}(\Omega)}<\infty$. Let us show now that $\sup _{\epsilon}\left\|\frac{v_{\epsilon}}{d}\right\|_{L^{\infty}(\Omega)}<\infty$. For this, we just need to estimate $v_{\epsilon}$ near the boundary. Set $\bar{v}_{\epsilon}$ the unique solution to

$$
\left\{\begin{array}{l}
-\Delta_{p}\left(u_{\lambda}+\bar{v}_{\epsilon}\right)+\mu_{\epsilon} \Delta_{p} \bar{v}_{\epsilon}=\frac{\lambda}{\left(\max \left\{\underline{u}_{\lambda}, u_{\lambda}+\bar{v}_{\epsilon}\right\}\right)^{\delta}} \text { in } \Omega ; \\
\bar{v}_{\epsilon}=0 \text { on } \partial \Omega .
\end{array}\right.
$$

Observing that $(\eta-1) u_{\lambda}$ is a subsolution to (3.27) for $\eta>0$ small enough and $K u_{\lambda}$ is a supersolution for $K>0$ large, we get $(\eta-1) u_{\lambda} \leq \bar{v}_{\epsilon} \leq K u_{\lambda}, \eta$ and $K$ could be chosen independently of $\epsilon$. Furthermore, $\bar{v}_{\epsilon} \leq v_{\epsilon}$. Indeed,

$$
\begin{aligned}
0 \leq & \int_{\bar{v}_{\epsilon}-v_{\epsilon}>0}\left(-\Delta_{p}\left(u_{\lambda}+\bar{v}_{\epsilon}\right)+\Delta_{p}\left(u_{\lambda}+v_{\epsilon}\right)\right)\left(\bar{v}_{\epsilon}-v_{\epsilon}\right) \mathrm{d} x \\
& +\mu_{\epsilon} \int_{\bar{v}_{\epsilon}-v_{\epsilon}>0}\left(-\Delta_{p} \bar{v}_{\epsilon}+\Delta_{p} v_{\epsilon}\right)\left(\bar{v}_{\epsilon}-v_{\epsilon}\right) \mathrm{d} x \\
\leq & \int_{\bar{v}_{\epsilon}-v_{\epsilon}>0}\left(\frac{\lambda}{\max \left\{\underline{u}_{\lambda}, u_{\lambda}+\bar{v}_{\epsilon}\right\}^{\delta}}-\frac{\lambda}{\max \left\{\underline{u}_{\lambda}, u_{\lambda}+v_{\epsilon}\right\}^{\delta}}\right)\left(\bar{v}_{\epsilon}-v_{\epsilon}\right) \mathrm{d} x \leq 0
\end{aligned}
$$


from which it follows that $\bar{v}_{\epsilon} \leq v_{\epsilon}$ and $\eta u_{\lambda} \leq u_{\lambda}+v_{\epsilon}$. Finally, using the weak comparison principle in a small neighborhood of $\partial \Omega$ and for $K$ large enough (independent of $\epsilon$ ), we get $v_{\epsilon} \leq K u_{\lambda}$ near the boundary. Hence, we have $\sup _{\epsilon}\left\|\frac{v_{\epsilon}}{d}\right\|_{L^{\infty}(\Omega)}<$ $\infty$. Now, using Theorem B.1 in Appendix B, it follows that for some $0<\beta<1$, $\sup _{\epsilon}\left\|v_{\epsilon}\right\|_{C^{1, \beta}(\bar{\Omega})}<\infty$. From Ascoli-Arzela theorem, we get then $v_{\epsilon} \rightarrow 0$ as $\epsilon \rightarrow 0^{+}$in $C^{1}(\bar{\Omega})$ which contradicts the definition of $v_{\epsilon}$ since $u_{\lambda}$ is a $C^{1}$-minimizer of $\underline{E}_{\lambda}, u_{\lambda}>0$ in $\Omega$ and $\frac{\partial u_{\lambda}}{\partial v}<0$ on $\partial \Omega$. This completes the proof of Proposition 3.7 in the subcritical case. Now, we deal with the critical case, i.e. $q=p^{*}-1$. Following some ideas contained in Brezis and Nirenberg [12], we use the truncated nonlinearity $f_{\lambda, j}: \bar{\Omega} \times \mathbb{R} \rightarrow \mathbb{R}^{+}$defined by

$$
f_{\lambda, j}(x, s) \stackrel{\text { def }}{=} \lambda \max \left\{\underline{u}_{\lambda}(x), s\right\}^{-\delta}+\min \left\{\max \left\{\underline{u}_{\lambda}(x), s\right\}^{q}, j^{q}\right\} .
$$

Let $F_{\lambda, j}(x, s)=\int_{0}^{s} f_{\lambda, j}(x, t) \mathrm{d} t$. Define $\underline{E}_{\lambda, j}: W_{0}^{1, p}(\Omega) \rightarrow \mathbb{R}$ by

$$
\underline{E}_{\lambda, j}(u) \stackrel{\text { def }}{=} \frac{1}{p} \int_{\Omega}|\nabla u|^{p} \mathrm{~d} x-\int_{\Omega} F_{\lambda, j}(x, u) \mathrm{d} x .
$$

Then, $\underline{E}_{\lambda, j}$ is weakly lower semicontinuous on $W_{0}^{1, p}(\Omega)$ and

$$
\forall w \in W_{0}^{1, p}(\Omega), \quad \underline{E}_{\lambda, j}(w) \rightarrow \underline{E}_{\lambda}(w) \quad \text { as } j \rightarrow \infty .
$$

Now, suppose that $u_{\lambda}$ is not a local minimizer of $\underline{E}_{\lambda}$ in $W_{0}^{1, p}(\Omega)$. Then, for any $\epsilon>0$ small enough, there exists $v_{\epsilon} \in W_{0}^{1, p}(\Omega)$ such that $\left\|v_{\epsilon}\right\|_{W_{0}^{1, p}(\Omega)} \leq \epsilon$ and

$$
\underline{E}_{\lambda}\left(u_{\lambda}+v_{\epsilon}\right)<\underline{E}_{\lambda}\left(u_{\lambda}\right)
$$

From (3.29) and (3.30), there exists $j(\epsilon) \in \mathbb{N}$ such that $j(\epsilon) \rightarrow \infty$ as $\epsilon \rightarrow 0^{+}$and satisfying

$$
\underline{E}_{\lambda, j(\epsilon)}\left(u_{\lambda}+v_{\epsilon}\right)<\underline{E}_{\lambda}\left(u_{\lambda}\right)=\underline{E}_{\lambda, j(\epsilon)}\left(u_{\lambda}\right) .
$$

Therefore, for any $\epsilon>0$ small enough, there exists $w_{\epsilon} \in W_{0}^{1, p}(\Omega)$ such that $\left\|w_{\epsilon}\right\|_{W_{0}^{1, p}(\Omega)} \leq \epsilon$ and satisfying

$$
\underline{E}_{\lambda, j(\epsilon)}\left(u_{\lambda}+w_{\epsilon}\right)=\min _{\|v\|_{W_{0}^{1, p}(\Omega)} \leq \epsilon} \underline{E}_{\lambda, j(\epsilon)}\left(u_{\lambda}+v\right) \leq \underline{E}_{\lambda, j(\epsilon)}\left(u_{\lambda}+v_{\epsilon}\right) .
$$

As in the subcritical case, we can prove that there exists $\mu_{\epsilon} \leq 0$ such that

$$
-\Delta_{p}\left(u_{\lambda}+w_{\epsilon}\right)-f_{\lambda, j(\epsilon)}\left(x, u_{\lambda}+w_{\epsilon}\right)=-\mu_{\epsilon} \Delta_{p} w_{\epsilon} .
$$

As above, we get that $\sup _{\epsilon}\left\|w_{\epsilon}\right\|_{C^{1, \beta}(\bar{\Omega})}<\infty$ for some $0<\beta<1$. Hence, $w_{\epsilon} \rightarrow 0$ in $C^{1}(\bar{\Omega})$ as $\epsilon \rightarrow 0^{+}$. Together with (3.31) and (3.32), it implies that for $\epsilon>0$ small enough

$$
\underline{E}_{\lambda, j(\epsilon)}\left(u_{\lambda}+w_{\epsilon}\right)=\underline{E}_{\lambda}\left(u_{\lambda}+w_{\epsilon}\right)<\underline{E}_{\lambda}\left(u_{\lambda}\right)
$$

which contradicts the fact that $u_{\lambda}$ is a $C^{1}$ local minimizer of $\underline{E}_{\lambda}$. The proof of Proposition 3.7 is now complete. 


\section{Existence of a second weak solution for $0<\lambda<\Lambda$}

Now, we are able to show the existence of a second solution using the Moutain Pass lemma. As in Paragraph 3.1, since the functional $E_{\lambda}$ is not $C^{1}$, we use the cut-off functional $\underline{E}_{\lambda}$ defined in (3.5). We recall that from Proposition 3.7, $u_{\lambda}$, given by Proposition 3.4, is a local minimizer of $\underline{E}_{\lambda}$. Moreover, from Theorem 2.3, any critical point $v_{\lambda}$ of $\underline{E}_{\lambda}$ satisfies $v_{\lambda}>\underline{u}_{\lambda}$ in $\Omega$ and hence solves (P). Therefore, to prove the existence of a second solution it is enough to show that $\underline{E}_{\lambda}$ has a critical point $v_{\lambda}$ different from $u_{\lambda}$. We first define a generalized notion of Palais Smale sequence for $\underline{E}_{\lambda}$ :

Definition 4.1. Let $\mathcal{F} \subset W_{0}^{1, p}(\Omega)$ be a closed set. We say that a sequence $\left\{v_{n}\right\}_{n=1}^{\infty} \subset$ $W_{0}^{1, p}(\Omega)$ is a Palais Smale sequence for $\underline{E}_{\lambda}$ at the level $c$ around $\mathcal{F}\left(\right.$ a $\left(P S_{\mathcal{F}, c}\right)$ for short) if

$$
\lim _{n \rightarrow \infty} \operatorname{dist}\left(v_{n}, \mathcal{F}\right)=0, \quad \lim _{n \rightarrow \infty} \underline{E}_{\lambda}\left(v_{n}\right)=c, \quad \lim _{n \rightarrow \infty}\left\|\underline{E}_{\lambda}^{\prime}\left(v_{n}\right)\right\|_{W^{-1, p^{\prime}}(\Omega)}=0 .
$$

We have the following compactness result for $\left(P S_{\mathcal{F}, c}\right)$ sequences for $\underline{E}_{\lambda}$ :

Lemma 4.2. Let $\mathcal{F} \subset W_{0}^{1, p}(\Omega)$ be a closed set, $c \in \mathbb{R}$. Let $\left\{v_{n}\right\}_{n=1}^{\infty} \subset W_{0}^{1, p}(\Omega)$ be a $\left(P S_{\mathcal{F}, c}\right)$ sequence for $\underline{E}_{\lambda}$. Then $\left\{v_{n}\right\}_{n=1}^{\infty}$ is bounded in $W_{0}^{1, p}(\Omega)$ and there exists a subsequence of $\left\{v_{n}\right\}_{n=1}^{\infty}$, we still denote by $\left\{v_{n}\right\}_{n=1}^{\infty}$, such that $v_{n} \rightarrow v_{\lambda}$ in $W_{0}^{1, p}(\Omega)$ where $v_{\lambda}$ is a weak solution to $(\mathrm{P})$.

Proof. From Definition 4.1, there exists $K>0$ such that

$$
\begin{aligned}
& \frac{1}{p} \int_{\Omega}\left|\nabla v_{n}\right|^{p} \mathrm{~d} x \\
& \quad-\int_{v_{n}>\underline{u}_{\lambda}}\left[\left(\frac{\lambda}{1-\delta} v_{n}^{1-\delta}+\frac{v_{n}^{q+1}}{q+1}\right)-\left(\frac{\lambda}{1-\delta} \underline{u}_{\lambda}^{1-\delta}+\frac{\underline{u}_{\lambda}^{q+1}}{q+1}\right)\right] \mathrm{d} x \\
& \quad-\int_{v_{n} \leq \underline{u}_{\lambda}} v_{n}\left(\lambda \underline{u}_{\lambda}^{-\delta}+\underline{u}_{\lambda}^{q}\right) \mathrm{d} x \leq K
\end{aligned}
$$

from which it follows that

$$
\frac{1}{p} \int_{\Omega}\left|\nabla v_{n}\right|^{p} \mathrm{~d} x-\int_{v_{n}>\underline{u}_{\lambda}}\left(\frac{\lambda}{1-\delta} v_{n}^{1-\delta}+\frac{v_{n}^{q+1}}{q+1}\right) \mathrm{d} x \leq K .
$$

Again from Definition 4.1 we have

$$
\begin{aligned}
\int_{\Omega}\left|\nabla v_{n}\right|^{p} \mathrm{~d} x= & \int_{v_{n}>\underline{u}_{\lambda}}\left(\lambda v_{n}^{1-\delta}+v_{n}^{q+1}\right) \mathrm{d} x+\int_{v_{n} \leq \underline{u}_{\lambda}}\left(\lambda \underline{u}_{\lambda}^{-\delta}+\underline{u}_{\lambda}^{q}\right) v_{n} \mathrm{~d} x \\
& +o_{n}(1)\left\|v_{n}\right\|_{W_{0}^{1, p}(\Omega)} .
\end{aligned}
$$


From (4.1) and (4.2) we get

$$
\begin{aligned}
& \left\|v_{n}\right\|_{W_{0}^{1, p}(\Omega)}^{p}+O_{n}\left(\left\|v_{n}\right\|_{W_{0}^{1, p}(\Omega)}\right) \\
& \geq \int_{v_{n}>\underline{u}_{\lambda}} v_{n}^{q+1} \mathrm{~d} x \geq \frac{q+1}{p}\left\|v_{n}\right\|_{W_{0}^{1, p}(\Omega)}^{p}-K .
\end{aligned}
$$

From (4.3), it follows that $\left\{v_{n}\right\}_{n=1}^{\infty}$ is bounded in $W_{0}^{1, p}(\Omega)$ and there exists $v_{\lambda}$ such that a suitable subsequence satisfies $v_{n} \rightarrow v_{\lambda}$ in $W_{0}^{1, p}(\Omega)$. Let $\phi \in W_{0}^{1, p}(\Omega)$. From Definition 4.1, we get

$$
\int_{\Omega}\left|\nabla v_{n}\right|^{p-2} \nabla v_{n} \nabla \phi \mathrm{d} x=\int_{\Omega} f_{\lambda}\left(x, v_{n}\right) \phi \mathrm{d} x+o_{n}(1) .
$$

Since $f_{\lambda}\left(x, v_{n}\right) \leq \underline{u}_{\lambda}^{-\delta}+\left(\max \left(\underline{u}_{\lambda}, v_{n}\right)\right)^{q}, q \leq p^{*}-1, v_{n} \rightarrow v_{\lambda}$ in $W_{0}^{1, p}(\Omega)$ and doing $n \rightarrow \infty$ in (4.4), we get

$$
\int_{\Omega}\left|\nabla v_{\lambda}\right|^{p-2} \nabla v_{\lambda} \nabla \phi \mathrm{d} x=\int_{\Omega} f_{\lambda}\left(x, v_{\lambda}\right) \phi \mathrm{d} x .
$$

From Theorem 2.3 and (4.5), it follows that $v_{\lambda}$ is a weak solution to (P). This completes the proof of Lemma 4.2.

We observe that from Proposition 3.4 and the fact that $\lim _{t \rightarrow \infty} \underline{E}_{\lambda}(t \phi)=-\infty$ for $0 \leq \phi \in W_{0}^{1, p}(\Omega) \backslash\{0\}, \underline{E}_{\lambda}$ has a Moutain Pass geometry close to $u_{\lambda}$. Hence we may fix $e \in W_{0}^{1, p}(\Omega) \backslash\{0\}$ such that $\underline{E}_{\lambda}(e)<\underline{E}_{\lambda}\left(u_{\lambda}\right)$. Let $R_{0}=\left\|e-u_{\lambda}\right\|_{W_{0}^{1, p}(\Omega)}$, $l_{0}>0$ small enough such that $u_{\lambda}$ is a minimizer of $\underline{E}_{\lambda}$ on $\overline{\mathcal{B}_{l_{0}}\left(u_{\lambda}\right)}$. Set

$$
\Gamma \stackrel{\text { def }}{=}\left\{\eta \in C\left([0,1], W_{0}^{1, p}(\Omega)\right) \mid \eta(0)=u_{\lambda}, \eta(1)=e\right\}
$$

and define the mountain pass level

$$
\gamma_{0}=\inf _{\eta \in \Gamma} \max _{t \in[0,1]} E_{\lambda}(\eta(t))
$$

We distinguish between the following two cases:

(P1) "Zero altitude case"

$$
\inf \left\{\underline{E}_{\lambda}(u): u \in W_{0}^{1, p}(\Omega) \text { and }\left\|u-u_{\lambda}\right\|_{W_{0}^{1, p}(\Omega)}=l\right\} \leq \underline{E}_{\lambda}\left(u_{\lambda}\right)
$$

for all $l<R_{0}$;

(P2) there exists $l_{1}<R_{0}$ such that

$$
\inf \left\{\underline{E}_{\lambda}(u): u \in W_{0}^{1, p}(\Omega) \text { and }\left\|u-u_{\lambda}\right\|_{W_{0}^{1, p}(\Omega)}=l\right\}>\underline{E}_{\lambda}\left(u_{\lambda}\right) \text {. }
$$


Note that (P1) (respectively (P2)) implies that $\gamma_{0}=\underline{E}_{\lambda}\left(u_{\lambda}\right)$ (respectively $\gamma_{0}>$ $\left.\underline{E}_{\lambda}\left(u_{\lambda}\right)\right)$. In case where $(\mathrm{P} 1)$ occurs, we can construct a $\left(P S_{\mathcal{F}, \gamma_{0}}\right)$ sequence with $\mathcal{F}=\partial \mathcal{B}_{l}\left(u_{\lambda}\right), l \leq l_{0}$, and get at least a second weak solution to $(\mathrm{P})$. More precisely, we have the following result:

Proposition 4.3. Let $p \in(1, \infty), q \in\left(p-1, p^{*}-1\right], \delta \in(0,1)$ and $\lambda \in(0, \Lambda)$. Suppose that $(\mathrm{P} 1)$ holds. Then, there exists a weak solution $v_{\lambda}$ of $(\mathrm{P})$ such that $v_{\lambda} \neq u_{\lambda}$.

Proof. From Theorem (1) in Ghoussoub and Preiss [25], for $l \leq l_{0}$ we get the existence of a $\left(P S_{\mathcal{F}, \gamma_{0}}\right)$ sequence, $\left\{v_{k}\right\}_{k=1}^{\infty}$. From Lemma $4.2,\left\{v_{k}\right\}_{k=1}^{\infty}$ is bounded and up to a subsequence weakly convergent in $W_{0}^{1, p}(\Omega)$ to $v_{\lambda}$, a weak solution to (P). To prove that $u_{\lambda} \neq v_{\lambda}$, it is sufficient to prove that $v_{k} \rightarrow v_{\lambda}$ strongly in $W_{0}^{1, p}(\Omega)$ as $k \rightarrow \infty$. Since $v_{k} \rightarrow v_{\lambda}$ as $k \rightarrow \infty$, applying Theorem 2.1 in Boccardo and Murat [9] as in the proof of Lemma 3.3 and from Remark 2.1 in [9], we get the following result from Brezis-Lieb (see [10]): As $k \rightarrow \infty$,

$$
\begin{aligned}
& \left\|v_{k}\right\|_{W_{0}^{1, p}(\Omega)}=\left\|v_{k}-v_{\lambda}\right\|_{W_{0}^{1, p}(\Omega)}+\left\|v_{\lambda}\right\|_{W_{0}^{1, p}(\Omega)}+o_{k}(1) \text { and } \\
& \left\|v_{k}\right\|_{L^{q+1}(\Omega)}=\left\|v_{k}-v_{\lambda}\right\|_{L^{q+1}(\Omega)}+\left\|v_{\lambda}\right\|_{L^{q+1}(\Omega)}+o_{k}(1) .
\end{aligned}
$$

By Sobolev imbedding theorem, we have also:

$$
\int_{v_{k} \geq \underline{u}_{\lambda}}\left|v_{k}^{1-\delta}-v_{\lambda}^{1-\delta}\right| \mathrm{d} x=o_{k}(1) \text { as } k \rightarrow \infty .
$$

Since $v_{\lambda}$ is a weak solution to $(\mathrm{P})$, we have:

$$
\left\|v_{\lambda}\right\|_{W_{0}^{1, p}(\Omega)}^{p}-\left\|v_{\lambda}\right\|_{L^{q+1}(\Omega)}^{q+1}-\lambda \int_{\Omega} v_{\lambda}^{1-\delta}=0 .
$$

Therefore, as $k \rightarrow \infty$

$$
\begin{aligned}
\int_{\Omega}\left|\nabla v_{k}\right|^{p-2} \nabla v_{k} \nabla\left(v_{k}-v_{\lambda}\right) \mathrm{d} x= & \lambda \int_{v_{k} \geq \underline{u}_{\lambda}} v_{k}^{-\delta}\left(v_{k}-v_{\lambda}\right) \mathrm{d} x \\
& +\int_{\Omega} v_{k}^{q}\left(v_{k}-v_{\lambda}\right) \mathrm{d} x+o_{k}(1) .
\end{aligned}
$$

It follows from (4.6), (4.8) and (4.7) that

$$
\int_{\Omega}\left|\nabla v_{k}-\nabla v_{\lambda}\right|^{p} \mathrm{~d} x=\int_{\Omega}\left|v_{k}-v_{\lambda}\right|^{q+1} \mathrm{~d} x+o_{k}(1) \text { as } k \rightarrow \infty .
$$

Now, we consider two cases:

(i) $\underline{E}_{\lambda}\left(u_{\lambda}\right) \neq \underline{E}_{\lambda}\left(v_{\lambda}\right)$,

(ii) $\underline{E}_{\lambda}\left(u_{\lambda}\right)=\underline{E}_{\lambda}\left(v_{\lambda}\right)$. 
In case (i), we are done. If (ii) holds, then from (4.6), we get

$$
\underline{E}_{\lambda}\left(v_{k}-v_{\lambda}\right)=\underline{E}_{\lambda}\left(v_{k}\right)-\underline{E}_{\lambda}\left(v_{\lambda}\right)+o_{k}(1)=o_{k}(1) \text { as } k \rightarrow \infty .
$$

Thus,

$$
\frac{1}{p}\left\|v_{k}-v_{\lambda}\right\|_{W_{0}^{1, p}(\Omega)}^{p}-\frac{1}{q+1}\left\|v_{k}-v_{\lambda}\right\|_{L^{q+1}(\Omega)}^{q+1} \leq o_{k}(1) \text { as } k \rightarrow \infty .
$$

Then, from (4.9) and (4.10), we obtain $\left\|v_{k}-v_{\lambda}\right\|_{W_{0}^{1, p}(\Omega)} \rightarrow 0$ as $k \rightarrow \infty$. Hence $\left\|u_{\lambda}-v_{\lambda}\right\|_{W_{0}^{1, p}(\Omega)}=l$ and $u_{\lambda} \neq v_{\lambda}$. This completes the proof of Proposition 4.3.

In case where (P2) occurs, we have the following result:

Proposition 4.4. Let the pair $(p, q)$ satisfy either $p \in(1, \infty)$ and $q \in\left(p-1, p^{*}-\right.$ $1)$, or else $p \in\left(\frac{2 N}{N+2}, 2\right] \cup\left(\frac{3 N}{N+3}, 3\right)$ and $q=p^{*}-1$. Let $\lambda \in(0, \Lambda)$ and suppose that $(\mathrm{P} 2)$ holds. Then there exists a weak solution $v_{\lambda}$ such that $u_{\lambda} \neq v_{\lambda}$.

Proof. We give the proof only in the second case (critical case) i.e. $p \in\left(\frac{2 N}{N+2}, 2\right] \cup$ $\left(\frac{3 N}{N+3}, 3\right)$ and $q=p^{*}-1$. The first case (subcritical case) i.e. $p \in(1, \infty)$ and $q \in\left(p-1, p^{*}-1\right)$ follows from Lemma 4.2 and Lemma C.1 in Appendix C. First, without loss of generality, we can assume that $u_{\lambda}$ has the minimal energy among all weak solutions (if not, we would have already found our second solution). Let $\mathcal{F}=W_{0}^{1, p}(\Omega)$. By Lemma 4.2, all $\left(P S_{\mathcal{F}, \gamma_{0}}\right)$ sequences are bounded in $W_{0}^{1, p}(\Omega)$. Again we need to prove the compactness of the $\left(P S_{\mathcal{F}, \gamma_{0}}\right)$ sequences in $W_{0}^{1, p}(\Omega)$. For that, we show that $\gamma_{0}$ is strictly below the first critical level where the PalaisSmale condition fails. Following the ideas in Brezis and Nirenberg [11], we use the test functions

$$
U_{\epsilon}(x)=\frac{C_{N} \epsilon^{\frac{N-p}{p(p-1)}}}{\left(\epsilon^{p /(p-1)}+|x-y|^{p /(p-1)}\right)^{\frac{N-p}{p}}} \phi(x)
$$

where $\epsilon>0, C_{N}$ is a normalization constant, $y \in \Omega$, and $\phi \in C_{\mathrm{c}}^{\infty}(\Omega)$ is a cut-off function such that $\phi=1$ in a neighborhood of $y$. Then, we prove the following statement:

Claim. There exists $\epsilon_{0}>0$ and $R_{0} \geq 1$ such that $\forall \epsilon \in\left(0, \epsilon_{0}\right)$

$$
\begin{aligned}
\underline{E}_{\lambda}\left(u_{\lambda}+R U_{\epsilon}\right) & =E_{\lambda}\left(u_{\lambda}+R U_{\epsilon}\right)<E_{\lambda}\left(u_{\lambda}\right) \forall R \geq R_{0}, \\
\underline{E}_{\lambda}\left(u_{\lambda}+t R_{0} U_{\epsilon}\right) & =\left(E_{\lambda}\left(u_{\lambda}+t R_{0} U_{\epsilon}\right)<E_{\lambda}\left(u_{\lambda}\right)+\frac{1}{N} S^{\frac{N}{p}} \forall t \in[0,1] .\right.
\end{aligned}
$$

Proof of the claim. The first inequality shows that $\Gamma$ is non empty. The proof is a direct consequence of the fact that $q>p-1$ and $R$ large. Let us prove the 
second inequality. We use the approach in García Azorero and Peral [20] where the following estimates are proved (see pages 946 and 949):

$$
\begin{aligned}
& \int_{\Omega}\left|\nabla u_{\lambda}+t R_{0} U_{\epsilon}\right|^{p} \mathrm{~d} x \leq \int_{\Omega}\left|\nabla u_{\lambda}\right|^{p} \mathrm{~d} x+\left(t R_{0}\right)^{p} \int_{\Omega}\left|\nabla U_{\epsilon}\right|^{p} \mathrm{~d} x \\
& \quad+p t R_{0} \int_{\Omega}\left|\nabla u_{\lambda}\right|^{p-2} \nabla u_{\lambda} \nabla U_{\epsilon} \mathrm{d} x+O\left(\epsilon^{\beta}\right)
\end{aligned}
$$

with $\beta>\frac{(N-p)}{p}$ and

$$
\begin{aligned}
& \int_{\Omega}\left(u_{\lambda}+t R_{0} U_{\epsilon}\right)^{q+1} \mathrm{~d} x \geq \int_{\Omega} u_{\lambda}^{q+1} \mathrm{~d} x+\left(t R_{0}\right)^{q+1} \int_{\Omega} U_{\epsilon}^{q+1} \mathrm{~d} x \\
& \quad+(q+1) t R_{0} \int_{\Omega} u_{\lambda}^{q} U_{\epsilon} \mathrm{d} x+(q+1)\left(t R_{0}\right)^{q} \int_{\Omega} u_{\lambda} U_{\epsilon}^{q} \mathrm{~d} x+O\left(\epsilon^{\gamma}\right)
\end{aligned}
$$

with $\gamma>\frac{(N-p)}{p}$. Then,

$$
\begin{aligned}
& E_{\lambda}\left(u_{\lambda}+t R_{0} U_{\epsilon}\right)=\frac{1}{p} \int_{\Omega}\left|\nabla u_{\lambda}+t R_{0} U_{\epsilon}\right|^{p} \mathrm{~d} x \\
& \quad-\frac{\lambda}{1-\delta} \int_{\Omega}\left(u_{\lambda}+t R_{0} U_{\epsilon}\right)^{1-\delta} \mathrm{d} x-\frac{1}{q+1} \int_{\Omega}\left(u_{\lambda}+t R_{0} U_{\epsilon}\right)^{q+1} \mathrm{~d} x \\
& \leq \int_{\Omega}\left|\nabla u_{\lambda}\right|^{p} \mathrm{~d} x+\frac{\left(t R_{0}\right)^{p}}{p} \int_{\Omega}\left|\nabla U_{\epsilon}\right|^{p} \mathrm{~d} x+t R_{0} \int_{\Omega}\left(\lambda u_{\lambda}^{-\delta}+u_{\lambda}^{q}\right) U_{\epsilon} \mathrm{d} x \\
& \quad-\frac{\lambda}{1-\delta} \int_{\Omega}\left(u_{\lambda}+t R_{0} U_{\epsilon}\right)^{1-\delta} \mathrm{d} x-\frac{1}{q+1} \int_{\Omega}\left(u_{\lambda}+t R_{0} U_{\epsilon}\right)^{q+1} \mathrm{~d} x+o\left(\epsilon \frac{N-p}{p}\right) \\
& \leq E_{\lambda}\left(u_{\lambda}\right)+\left(t R_{0}\right)^{p} \int_{\Omega}\left|\nabla U_{\epsilon}\right|^{p} \mathrm{~d} x-\frac{\left(t R_{0}\right)^{q+1}}{q+1} \int_{\Omega} U_{\epsilon}^{q+1} \mathrm{~d} x \\
& \quad-\left(t R_{0}\right)^{q} \int_{\Omega} u_{\lambda} U_{\epsilon}^{q} \mathrm{~d} x+t R_{0} \int_{\Omega} u_{\lambda}^{q} U_{\epsilon} \mathrm{d} x+\frac{\lambda}{1-\delta} \int_{\Omega} u_{\lambda}^{1-\delta} \mathrm{d} x \\
& \quad-\frac{\lambda}{1-\delta} \int_{\Omega}\left(u_{\lambda}+t R_{0} U_{\epsilon}\right)^{1-\delta} \mathrm{d} x+t R_{0} \int_{\Omega} \lambda u_{\lambda}^{-\delta} U_{\epsilon} \mathrm{d} x+o\left(\epsilon \frac{N-p}{p}\right) .
\end{aligned}
$$

Now, we estimate the last three terms as follows:

$$
\begin{aligned}
& \frac{\lambda}{1-\delta} \int_{\Omega} u_{\lambda}^{1-\delta} \mathrm{d} x-\frac{\lambda}{1-\delta} \int_{\Omega}\left(u_{\lambda}+t R_{0} U_{\epsilon}\right)^{1-\delta} \mathrm{d} x+t R_{0} \int_{\Omega} \lambda u_{\lambda}^{-\delta} U_{\epsilon} \mathrm{d} x \\
& \leq K \int_{B_{\mu}(y)} U_{\epsilon} \mathrm{d} x
\end{aligned}
$$

for $\mu>0$. Moreover,

$$
\int_{B_{\mu}(y)} U_{\epsilon} \mathrm{d} x \leq \begin{cases}\epsilon^{N-\frac{(N-p)}{p}}\left[O(1)+O\left(\epsilon^{\frac{N-p}{p-1}-N}\right)\right] & \text { if } p \neq \frac{2 N}{N+1} \\ O\left(\epsilon^{N-\frac{(N-p)}{p}} \log \epsilon\right) & \text { if } p=\frac{2 N}{N+1}\end{cases}
$$


From (4.14) and $p \in\left(\frac{2 N}{N+2}, 2\right)$, we get

$$
\int_{B_{\mu}(y)} U_{\epsilon} \mathrm{d} x=o\left(\epsilon^{\frac{N-p}{p}}\right) .
$$

Now, assume that $p \in\left(\frac{3 N}{N+3}, 3\right)$. In this case, using the Taylor expansion, we estimate the last three terms in (4.13) as follows:

$$
\begin{aligned}
& \frac{\lambda}{1-\delta} \int_{\Omega} u_{\lambda}^{1-\delta} \mathrm{d} x-\frac{\lambda}{1-\delta} \int_{\Omega}\left(u_{\lambda}+t R_{0} U_{\epsilon}\right)^{1-\delta} \mathrm{d} x+t R_{0} \int_{\Omega} \lambda u_{\lambda}^{-\delta} U_{\epsilon} \mathrm{d} x \\
& \leq K \int_{B_{\mu}(y)} U_{\epsilon}^{2} \mathrm{~d} x
\end{aligned}
$$

for $\mu>0$. Moreover,

$$
\int_{B_{\mu}(y)} U_{\epsilon}^{2} \mathrm{~d} x \leq\left\{\begin{array}{l}
\epsilon^{N-\frac{2(N-p)}{p}}\left[O(1)+O\left(\epsilon^{\frac{2(N-p)}{p-1}-N}\right)\right] \text { if } p \neq \frac{3 N}{N+2}, \\
O\left(\epsilon^{N-\frac{2(N-p)}{p}} \log \epsilon\right) \text { if } p=\frac{3 N}{N+2}
\end{array}\right.
$$

From (4.16) and $p \in\left(\frac{3 N}{N+3}, 3\right)$, we get

$$
\int_{B_{\mu}(y)} U_{\epsilon}^{2} \mathrm{~d} x=o\left(\epsilon^{\frac{N-p}{p}}\right) .
$$

Thus, From (4.13), (4.15) and (4.17), it follows that

$$
\begin{aligned}
E_{\lambda}\left(u_{\lambda}+t R_{0} U_{\epsilon}\right) \leq & E_{\lambda}\left(u_{\lambda}\right)+\left(t R_{0}\right)^{p} \int_{\Omega}\left|\nabla U_{\epsilon}\right|^{p} \mathrm{~d} x-\frac{\left(t R_{0}\right)^{q+1}}{q+1} \int_{\Omega} U_{\epsilon}^{q+1} \mathrm{~d} x \\
& -\left(t R_{0}\right)^{q} \int_{\Omega} u_{\lambda} U_{\epsilon}^{q} \mathrm{~d} x+o\left(\epsilon^{\frac{N-p}{p}}\right) .
\end{aligned}
$$

for $p \in\left(\frac{2 N}{N+2}, 2\right) \cup\left(\frac{3 N}{N+3}, 3\right)$. The case $p=2$ is done in Haitao [29] and in Hirano, Saccon and Shioji [31]. Arguing as in García Azorero and Peral [20] (see page 947), we get for $p \in\left(\frac{2 N}{N+2}, 2\right] \cup\left(\frac{3 N}{N+3}, 3\right)$ :

$$
\sup _{t \in \mathbb{R}^{+}} E_{\lambda}\left(u_{\lambda}+t R_{0} U_{\epsilon}\right)<E_{\lambda}\left(u_{\lambda}\right)+\frac{1}{N} S^{\frac{N}{p}}
$$

which completes the proof of the claim Now, the compactness of $\left\{v_{k}\right\}_{k=1}^{\infty}$ implies that $E_{\lambda}\left(v_{\lambda}\right)=\gamma_{0}>E_{\lambda}\left(u_{\lambda}\right)$. Therefore $v_{\lambda} \neq u_{\lambda}$.

Thus, the proof of Theorem 2.1 follows from Propositions 4.3 and 4.4. Now, Theorem 2.2 follows from the subsequent regularity results given in Appendices A and $\mathrm{B}$. 


\section{A. Appendix}

We start with an important technical tool which enables us to estimate the singularity in the Gâteaux derivative of the energy functional $E_{\lambda}: W_{0}^{1, p}(\Omega) \rightarrow \mathbb{R}$ defined in (1.1).

Lemma A.1. Let $0<\delta<1$. Then there exists a constant $C_{\delta}>0$ such that the inequality

$$
\int_{0}^{1}|\mathbf{a}+s \mathbf{b}|^{-\delta} \mathrm{d} s \leq C_{\delta}\left(\max _{0 \leq s \leq 1}|\mathbf{a}+s \mathbf{b}|\right)^{-\delta}
$$

holds true for all $\mathbf{a}, \mathbf{b} \in \mathbb{R}^{N}$ with $|\mathbf{a}|+|\mathbf{b}|>0$.

An elementary proof of this lemma can be found in Takáč [40, Lemma A.1, page 233].

We continue by showing the Gâteaux-differentiability of the energy functional $E_{\lambda}$ at a point $u \in W_{0}^{1, p}(\Omega)$ satisfying $u \geq \varepsilon \varphi_{1}$ in $\Omega$ with a constant $\varepsilon>0$.

Lemma A.2. Let $0<\delta<1,1<p<\infty$, and $p-1<q \leq p^{*}-1$. Assume that $u, v \in W_{0}^{1, p}(\Omega)$ and $u$ satisfies $u \geq \varepsilon \varphi_{1}$ in $\Omega$ with a constant $\varepsilon>0$. Then we have

$$
\begin{aligned}
& \lim _{t \rightarrow 0} \frac{1}{t}\left(E_{\lambda}(u+t v)-E_{\lambda}(u)\right) \\
& =\int_{\Omega}|\nabla u|^{p-2} \nabla u \cdot \nabla v \mathrm{~d} x-\lambda \int_{\Omega} u^{-\delta} v \mathrm{~d} x-\int_{\Omega} u^{q} v \mathrm{~d} x .
\end{aligned}
$$

Proof. We show the result only for the singular term $\int_{\Omega} u^{-\delta} v \mathrm{~d} x$; the other two terms are treated in a standard way. So let

$$
F(u)=\frac{1}{1-\delta} \int_{\Omega}\left(u^{+}\right)^{1-\delta} \mathrm{d} x \quad \text { for } u \in W_{0}^{1, p}(\Omega) .
$$

For $\xi \in \mathbb{R} \backslash\{0\}$ we define

$$
z(\xi)=\frac{1}{1-\delta} \frac{\mathrm{d}}{\mathrm{d} \xi}\left(\xi^{+}\right)^{1-\delta}=\left\{\begin{array}{cc}
\xi^{-\delta} & \text { if } \xi>0 \\
0 & \text { if } \xi<0
\end{array}\right.
$$

Consequently,

$$
\frac{1}{t}(F(u+t v)-F(u))=\int_{\Omega}\left(\int_{0}^{1} z(u+s t v) \mathrm{d} s\right) v \mathrm{~d} x .
$$

Notice that for almost every $x \in \Omega$ we have $u(x)>0$ and

$$
\int_{0}^{1} z(u(x)+\operatorname{stv}(x)) \mathrm{d} s \longrightarrow z(u(x))=u(x)^{-\delta} \quad \text { as } t \rightarrow 0 .
$$


Moreover, the integral on the left-hand side (with nonnegative integrand) is dominated by

$$
\begin{aligned}
\int_{0}^{1} z(u(x)+s t v(x)) \mathrm{d} s & \leq \int_{0}^{1}|u(x)+s t v(x)|^{-\delta} \mathrm{d} s \\
& \leq C_{\delta}\left(\max _{0 \leq s \leq 1}|u(x)+s t v(x)|\right)^{-\delta} \\
& \leq C_{\delta} u(x)^{-\delta} \leq C_{\delta}\left(\varepsilon \varphi_{1}(x)\right)^{-\delta}=C_{\delta, \varepsilon} \varphi_{1}(x)^{-\delta}
\end{aligned}
$$

with a constant $C_{\delta, \varepsilon}>0$ independent of $x \in \Omega$. Here, we have used the estimate (A.1) from Lemma A.1 above. Finally, we have $v \varphi_{1}^{-\delta} \in L^{1}(\Omega)$, by $v \in W_{0}^{1, p}(\Omega)$ and Hardy's inequality. Hence, we are allowed to invoke the Lebesgue dominated convergence theorem in (A.3) from which the lemma follows by letting $t \rightarrow 0$.

Corollary A.3. Let $0<\delta<1,1<p<\infty$, and $p-1<q \leq p^{*}-1$. Then the energy functional $E_{\lambda}: W_{0}^{1, p}(\Omega) \rightarrow \mathbb{R}$ is Gateaux-differentiable at every point $u \in W_{0}^{1, p}(\Omega)$ that satisfies $u \geq \varepsilon \varphi_{1}$ in $\Omega$ with a constant $\varepsilon>0$. Its Gâteaux derivative $E_{\lambda}^{\prime}(u)$ at $u$ is given by

$$
\begin{aligned}
& \left\langle E_{\lambda}^{\prime}(u), v\right\rangle= \\
& \int_{\Omega}|\nabla u|^{p-2} \nabla u \cdot \nabla v \mathrm{~d} x-\lambda \int_{\Omega} u^{-\delta} v \mathrm{~d} x-\int_{\Omega} u^{q} v \mathrm{~d} x
\end{aligned}
$$

for $v \in W_{0}^{1, p}(\Omega)$.

We continue by proving the $C^{1}$-differentiability of the cut off energy functional:

Lemma A.4. Let $0<\delta<1,1<p<\infty, p-1<q<\infty$, and $w \in W_{0}^{1, p}(\Omega)$ such that $w \geq \epsilon \varphi_{1}$ with some $\epsilon>0$. Setting for $x \in \Omega$

$$
\begin{aligned}
& f_{\lambda}(x, s)= \begin{cases}\lambda s^{-\delta}+s^{q} & \text { if } s \geq w(x), \\
\lambda w(x)^{-\delta}+w(x)^{q} & \text { if } s<w(x),\end{cases} \\
& F_{\lambda}(x, s)=\int_{0}^{s} f_{\lambda}(x, t) \mathrm{d} t \text { and for } u \in W_{0}^{1, p}(\Omega) \\
& \bar{E}_{\lambda}(u)=\frac{1}{p} \int_{\Omega}|\nabla u|^{p} \mathrm{~d} x-\int_{\Omega} F_{\lambda}(x, u) \mathrm{d} x,
\end{aligned}
$$

we have that $\bar{E}_{\lambda}$ belongs to $C^{1}\left(W_{0}^{1, p}(\Omega), \mathbb{R}\right)$. 
Proof. As in Lemma A.2, we concentrate on the singular term, the others being standard. Let

$$
h(x, s)= \begin{cases}s^{-\delta} & \text { if } s \geq w(x), \\ w(x)^{-\delta} & \text { if } s<w(x),\end{cases}
$$

$H(x, s)=\int_{0}^{s} h(x, t) \mathrm{d} t$, and $S(u)=\int_{\Omega} H(x, u) \mathrm{d} x$. Proceeding as in Lemma A.2, we obtain that for all $u \in W_{0}^{1, p}(\Omega), S(u)$ has a Gâteaux derivative $S^{\prime}(u)$ given by

$$
\left\langle S^{\prime}(u), v\right\rangle=\int_{\Omega}(\max \{u(x), w(x)\})^{-\delta} v(x) \mathrm{d} x .
$$

Let $u_{k} \in W_{0}^{1, p}(\Omega), u_{k} \rightarrow u_{0}$. Then

$$
\begin{aligned}
\left|\left\langle S^{\prime}\left(u_{k}\right)-S^{\prime}\left(u_{0}\right), v\right\rangle\right|= & \mid \int_{\Omega}\left(\left(\max \left\{u_{k}(x), w(x)\right\}\right)^{-\delta} v(x)\right. \\
& \left.-(\max \{u(x), w(x)\})^{-\delta} v(x)\right) \mathrm{d} x \mid \\
\leq & 2 \int_{\Omega} w^{-\delta}|v| \mathrm{d} x \\
\leq & 2 \epsilon^{-\delta} \int_{\Omega} \varphi_{1}^{-\delta}|v| \mathrm{d} x
\end{aligned}
$$

for all $v \in W_{0}^{1, p}(\Omega)$. Again, as in Lemma A.2, we use Hardy's inequality to deduce that $\varphi_{1}^{-\delta} v \in L^{1}$, so that by Lesbegue's dominated convergence theorem we conclude that the Gâteaux derivative of $S$ is continuous which implies that $S \in C^{1}\left(W_{0}^{1, p}(\Omega), \mathbb{R}\right)$.

Next, we give some regularity results for weak solutions to problem $(\mathrm{P})$. We start with the following lemma which allows for test functions $\phi$ in equation (2.1) to be taken in $W_{0}^{1, p}(\Omega)$ rather than only in $C_{\mathrm{c}}^{\infty}(\Omega)\left(\subset W_{0}^{1, p}(\Omega)\right)$.

Lemma A.5. Each positive weak solution $u$ of problem $(\mathrm{P})$ satisfies $u \geq \epsilon_{\lambda} \phi_{1}$ a.e. in $\Omega$, where $\epsilon_{\lambda}>0$ is a constant independent of $u$. Moreover, for every function $w \in W_{0}^{1, p}(\Omega)$ we have $u^{-\delta} w \in L^{1}(\Omega)$ and

$$
\int_{\Omega}|\nabla u|^{p-2} \nabla u \cdot \nabla w \mathrm{~d} x=\lambda \int_{\Omega} u^{-\delta} w \mathrm{~d} x+\int_{\Omega} u^{q} w \mathrm{~d} x .
$$

Proof. Let $u$ be a positive weak solution of (P). Recall that $u$ is required to satisfy

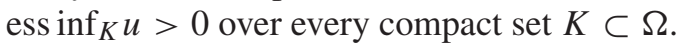

First, we establish the inequality

$$
\int_{\Omega}|\nabla u|^{p-2} \nabla u \cdot \nabla w \mathrm{~d} x \geq \lambda \int_{\Omega} u^{-\delta} w \mathrm{~d} x+\int_{\Omega} u^{q} w \mathrm{~d} x
$$


for every $w \in W_{0}^{1, p}(\Omega)$ satisfying $w \geq 0$ a.e. in $\Omega$. Given $0 \leq w \in W_{0}^{1, p}(\Omega)$, there exists a sequence $\left\{w_{k}\right\}_{k=1}^{\infty} \subset C_{\mathrm{c}}^{\infty}(\Omega)$ such that $w_{k} \geq 0$ in $\Omega$ and $w_{k} \rightarrow w$ strongly in $W_{0}^{1, p}(\Omega)$ as $k \rightarrow \infty$. Since $p<q+1 \leq p^{*}$, this entails $w_{k} \rightarrow w$ strongly also in $L^{q+1}(\Omega)$ as $k \rightarrow \infty$. Moreover, we can find a subsequence, denoted again by $\left\{w_{k}\right\}_{k=1}^{\infty}$, such that $w_{k} \rightarrow w$ almost everywhere in $\Omega$ as $k \rightarrow \infty$. In equation (A.5) we now replace $w$ by $w_{k}$ and apply Fatou's lemma to the integral $\int_{\Omega} u^{-\delta} w_{k} \mathrm{~d} x$ as $k \rightarrow \infty$, thus arriving at the desired inequality (A.6).

In particular, inequality (A.6) implies

$$
\int_{\Omega}|\nabla u|^{p-2} \nabla u \cdot \nabla w \mathrm{~d} x \geq \lambda \int_{\Omega} u^{-\delta} w \mathrm{~d} x
$$

whenever $0 \leq w \in W_{0}^{1, p}(\Omega)$. Now we are ready to compare $u$ with the unique weak solution $\underline{u}_{\lambda}$ of problem (3.1) obtained in Lemma 3.1. We apply the weak comparison principle ( $c f$. the proof of Theorem 2.3) to (the weak formulation of) problem (3.1) (with $\underline{u}_{\lambda}$ in place of $u$ ) and to inequality (A.7) (with $u$ ), thus obtaining $u \geq \underline{u}_{\lambda}$ a.e. in $\Omega$. This guarantees $u \geq \epsilon_{\lambda} \phi_{1}$ a.e. in $\Omega$.

Next, there are constants $0<\ell<L<\infty$ such that $\ell d(x) \leq \phi_{1}(x) \leq L d(x)$ for all $x \in \Omega$. It follows that $u \geq \epsilon_{\lambda} \ell d$ a.e. in $\Omega$. Now, instead of using Fatou's lemma in the limiting process above, we apply Hardy's inequality to the integral $\int_{\Omega} u^{-\delta} w_{k} \mathrm{~d} x$ as $k \rightarrow \infty$, thus arriving at the desired equality (A.5) for every $w \in$ $W_{0}^{1, p}(\Omega)$ satisfying $w \geq 0$ a.e. in $\Omega$.

Finally, we make use of the polar decomposition $w=w^{+}-w^{-}$of an arbitrary function $w \in W_{0}^{1, p}(\Omega)$, where $w^{+}=\max \{w, 0\}$ and $w^{-}=\max \{-w, 0\}$ satisfy $w^{+}, w^{-} \in W_{0}^{1, p}(\Omega)$ and $\nabla w=\nabla w^{+}-\nabla w^{-}$. Since we have already verified equation (A.5) for $w^{+}$and $w^{-}$, the desired equality (A.5) holds also for every $w \in$ $W_{0}^{1, p}(\Omega)$.

Lemma A.6. Each positive weak solution u of $(\mathrm{P})$ belongs to $L^{\infty}(\Omega)$.

Proof. First, we show that each positive weak solution $u$ of $(\mathrm{P})$ satisfies

$$
\int_{\Omega}\left|\nabla(u-1)^{+}\right|^{p-2} \nabla(u-1)^{+} \cdot \nabla w \mathrm{~d} x \leq \int_{\Omega}\left(\lambda+u^{q}\right) w \mathrm{~d} x
$$

for every $w \in C_{\mathrm{c}}^{\infty}(\Omega)$ with $w \geq 0$. Indeed, let $\psi: \mathbb{R} \rightarrow[0,1]$ be a $C^{1}$ cut-off function such that $\psi(s)=0$ if $s \leq 0, \psi^{\prime}(s) \geq 0$ if $0 \leq s \leq 1$, and $\psi(s)=1$ if $s \geq 1$. Given any $\epsilon>0$, define $\psi_{\epsilon}(t) \stackrel{\text { def }}{=} \psi((t-1) / \epsilon)$ for $t \in \mathbb{R}$. Hence, $\psi_{\epsilon} \circ u \in W_{0}^{1, p}(\Omega)$ with $\nabla\left(\psi_{\epsilon} \circ u\right)=\left(\psi_{\epsilon}^{\prime} \circ u\right) \nabla u$. Using the weak form of problem (P), equation (2.1), with the test function $\phi=\left(\psi_{\epsilon} \circ u\right) w$, where $w \in C_{\mathrm{c}}^{\infty}(\Omega)$ satisfies $w \geq 0$, we obtain

$$
\int_{\Omega}|\nabla u|^{p-2} \nabla u \cdot \nabla\left[\left(\psi_{\epsilon} \circ u\right) w\right] \mathrm{d} x=\int_{\Omega}\left(\lambda u^{-\delta}+u^{q}\right)\left(\psi_{\epsilon} \circ u\right) w \mathrm{~d} x .
$$


Hence,

$$
\begin{aligned}
& \int_{\Omega}|\nabla u|^{p}\left(\psi_{\epsilon}^{\prime} \circ u\right) w \mathrm{~d} x+\int_{\Omega}|\nabla u|^{p-2}(\nabla u \cdot \nabla w)\left(\psi_{\epsilon} \circ u\right) \mathrm{d} x \\
& =\int_{\Omega}\left(\lambda u^{-\delta}+u^{q}\right)\left(\psi_{\epsilon} \circ u\right) w \mathrm{~d} x
\end{aligned}
$$

with $\psi_{\epsilon}^{\prime} \circ u \geq 0$, which yields

$$
\int_{\Omega}|\nabla u|^{p-2}(\nabla u \cdot \nabla w)\left(\psi_{\epsilon} \circ u\right) \mathrm{d} x \leq \int_{\Omega}\left(\lambda u^{-\delta}+u^{q}\right)\left(\psi_{\epsilon} \circ u\right) w \mathrm{~d} x .
$$

Letting $\epsilon \rightarrow 0+$ we arrive at (A.8). Finally, the $L^{\infty}$ bound and regularity of $u$ are obtained directly from equation (A.8) as follows: if $q<p^{*}-1$, one applies Theorem A.1 from Anane [6], and if $q=p^{*}-1$, the bootstrapping arguments from the proof of Theorem A.1, pages 950-953, in García Azorero and Peral [20] yield the desired result. In both references $[6,20]$ the bootstrapping arguments use the technique due to Serrin [39] (proof of Theorem 1).

Finally, we are ready to bound any weak solution $u$ of problem $(\mathrm{P})$ by a positive scalar multiple of the eigenfunction $\phi_{1}$ also from above. This result complements the corresponding bound from below, $u \geq \epsilon_{\lambda} \phi_{1}$ a.e. in $\Omega$, stated in the first part of Lemma A.5 above. Equivalently, these lower and upper bounds for $u / \phi_{1}$ can be reformulated as follows, using the distance function $d$ in place of $\phi_{1}$ :

Lemma A.7. Each positive weak solution u of problem $(\mathrm{P})$ satisfies $c_{\lambda} d \leq u \leq$ $K_{\lambda} d$ a.e. in $\Omega$, where $0<c_{\lambda} \leq K_{\lambda}<\infty$ are some constants independent of $u$.

Proof. Let $u \in W_{0}^{1, p}(\Omega)$ be a positive weak solution of problem (P). It follows from the first part of Lemma A.5 and its proof that $u(x) \geq \underline{u}_{\lambda}(x) \geq \epsilon_{\lambda} \phi_{1}(x) \geq \epsilon_{\lambda} \ell d(x)$ for a.e. $x \in \Omega$. Hence, we can take $c_{\lambda}=\epsilon_{\lambda} \ell>0$ to get $u \geq c_{\lambda} d$ a.e. in $\Omega$.

Next, we take advantage of the inequality $u \geq c_{\lambda} d$ to derive also $u \leq K_{\lambda} d$. Recall that $u \in L^{\infty}(\Omega)$, by Lemma A.6 above. First, we apply the estimate

$$
u^{q}=\frac{u^{q+\delta}}{u^{\delta}} \leq \frac{\|u\|_{L^{\infty}(\Omega)}^{q+\delta}}{u^{\delta}} \quad \text { a.e. in } \Omega
$$

to the right-hand side of the equation in problem $(\mathrm{P})$ to conclude that

$$
\begin{cases}-\Delta_{p} u \leq\left(\lambda+\|u\|_{L^{\infty}(\Omega)}^{q+\delta}\right) u^{-\delta} & \text { in } \Omega \\ \left.u\right|_{\partial \Omega}=0, \quad u>0 & \text { in } \Omega\end{cases}
$$

After the substitution

$$
v=\left(1+\lambda^{-1}\|u\|_{L^{\infty}(\Omega)}^{q+\delta}\right)^{-1 /(p-1+\delta)} u,
$$


inequality (A.9) is equivalent with

$$
\begin{cases}-\Delta_{p} v \leq \lambda v^{-\delta} & \text { in } \Omega \\ \left.v\right|_{\partial \Omega}=0, \quad v>0 & \text { in } \Omega\end{cases}
$$

Now, in analogy with the proof of Lemma A.5, we apply the weak comparison principle to problem (3.1) (with $\underline{u}_{\lambda}$ in place of $u$ ) and to inequality (A.10) (with $v$ ), thus arriving at $v \leq \underline{u}_{\lambda}$ a.e. in $\bar{\Omega}$. Thus, it remains to verify $\underline{u}_{\lambda} \leq c_{\lambda}^{\prime} d$ a.e. in $\Omega$, where $0<c_{\lambda}^{\prime}<\infty$ is a constant. This will imply $u \leq K_{\lambda} d$ a.e. in $\Omega$ with

$$
K_{\lambda}=c_{\lambda}^{\prime}\left(1+\lambda^{-1}\|u\|_{L^{\infty}(\Omega)}^{q+\delta}\right)^{1 /(p-1+\delta)}
$$

Thanks to $\ell d(x) \leq \phi_{1}(x) \leq L d(x)$ for all $x \in \Omega$, with some constants $0<\ell<L<\infty$, the inequality $\underline{u}_{\lambda} \leq c_{\lambda}^{\prime} d$ in $\Omega$ is equivalent to $\underline{u}_{\lambda} \leq c_{\lambda}^{\prime \prime} \phi_{1}$ in $\Omega$, where $0<c_{\lambda}^{\prime \prime}<\infty$ is a constant. We now construct a supersolution $w$ to problem (3.1) of the form $w=\beta \cdot \Theta_{\alpha} \circ \phi_{1}$ in $\Omega$. Here, $\alpha, \beta>0$ are suitable numbers and $\Theta_{\alpha}:\left[0, R_{\alpha}\right) \rightarrow \mathbb{R}_{+}$is a $C^{1}$ function (where $0<R_{\alpha}<\infty$ and $\left.\mathbb{R}_{+}=[0, \infty)\right)$ that satisfies the initial value problem

$$
\left\{\begin{array}{l}
-\frac{\mathrm{d}}{\mathrm{d} r}\left(\left|\Theta_{\alpha}^{\prime}(r)\right|^{p-2} \Theta_{\alpha}^{\prime}(r)\right)=\Theta_{\alpha}(r)^{-\delta}, \quad 0<r<R_{\alpha} \\
\Theta_{\alpha}(0)=0, \quad \Theta_{\alpha}^{\prime}(0)=\alpha>0 .
\end{array}\right.
$$

The endpoint $R_{\alpha}$ is defined to be the supremum of all numbers $s \in(0, \infty)$ such that $\Theta_{\alpha}^{\prime}(r)>0$ holds for all $r \in[0, s)$. We will see that $0<R_{\alpha}<\infty$ together with $\Theta_{\alpha}^{\prime}(r) \searrow 0$ as $r \nearrow R_{\alpha}$.

Making use of the transformation

$$
\left\{\begin{array}{l}
\Theta_{\alpha}(r)=\alpha^{\frac{p}{1-\delta}} \cdot \Theta_{1}\left(\alpha^{-\frac{p}{p-1+\delta}} r\right), \quad 0 \leq r \leq R_{\alpha} \\
R_{\alpha}=\alpha^{\frac{p}{p-1+\delta}} R_{1}
\end{array}\right.
$$

we conclude that it suffices to treat the case $\alpha=1$. Problem (A.11) with $\alpha=1$ has the first integral

$$
\left\{\begin{array}{l}
-\frac{p-1}{p}\left|\Theta_{1}^{\prime}(r)\right|^{p}-\frac{1}{1-\delta} \Theta_{1}(r)^{1-\delta}+C=0, \quad 0 \leq r<R_{1} ; \\
\Theta_{1}(0)=0, \quad \Theta_{1}^{\prime}(0)=1>0,
\end{array}\right.
$$

where the constant $C$ is given by $C=(p-1) / p$. There exists precisely one $C^{1}$ function $\Theta_{1}:\left[0, R_{1}\right) \rightarrow \mathbb{R}_{+}$that satisfies (A.13) together with $\Theta_{1}^{\prime}(r)>0$ for all $r \in\left[0, R_{1}\right)$; it is determined from

$$
\int_{0}^{\Theta_{1}(r)}\left(1-\frac{p}{(p-1)(1-\delta)} \theta^{1-\delta}\right)^{-1 / p} \mathrm{~d} \theta=r, \quad 0 \leq r<R_{1},
$$


where

$$
\begin{aligned}
R_{1} & =\int_{0}^{[(p-1)(1-\delta) / p]^{1 /(1-\delta)}}\left(1-\frac{p}{(p-1)(1-\delta)} \theta^{1-\delta}\right)^{-1 / p} \mathrm{~d} \theta \\
& =\left(\frac{(p-1)(1-\delta)}{p}\right)^{1 /(1-\delta)} \int_{0}^{1}\left(1-t^{1-\delta}\right)^{-1 / p} \mathrm{~d} t<\infty
\end{aligned}
$$

is the maximal number such that $\Theta_{1}^{\prime}(r)>0$ for all $r \in\left[0, R_{1}\right)$.

Let us first fix $\alpha>0$ large enough, such that $R_{\alpha}>M \stackrel{\text { def }}{=} \max \bar{\Omega} \phi_{1}$. In the following calculations we make use of equations (2.2) and (A.11) for $\phi_{1}$ and $\Theta_{\alpha}$, respectively. The function $w(x)=\beta \cdot \Theta_{\alpha}\left(\phi_{1}(x)\right)$ of $x \in \Omega$ satisfies

$$
\begin{aligned}
\nabla w(x) & =\beta \cdot \Theta_{\alpha}^{\prime}\left(\phi_{1}(x)\right) \nabla \phi_{1}(x), \\
|\nabla w(x)|^{p-2} \nabla w(x) & =\beta^{p-1}\left[\Theta_{\alpha}^{\prime}\left(\phi_{1}(x)\right)\right]^{p-1}\left|\nabla \phi_{1}(x)\right|^{p-2} \nabla \phi_{1}(x),
\end{aligned}
$$

whence

$$
\begin{aligned}
-\Delta_{p} w= & -\beta^{p-1}\left[\left(\left(\Theta_{\alpha}^{\prime}\right)^{p-1}\right)^{\prime} \circ \phi_{1}\right]\left|\nabla \phi_{1}\right|^{p} \\
& +\beta^{p-1}\left[\left(\left(\Theta_{\alpha}^{\prime}\right)^{p-1}\right) \circ \phi_{1}\right]\left(-\Delta_{p} \phi_{1}\right) \\
= & \beta^{p-1}\left(\Theta_{\alpha} \circ \phi_{1}\right)^{-\delta}\left|\nabla \phi_{1}\right|^{p} \\
& +\beta^{p-1} \lambda_{1}\left[\left(\left(\Theta_{\alpha}^{\prime}\right)^{p-1}\right) \circ \phi_{1}\right] \cdot \phi_{1}^{p-1} \\
= & \beta^{p-1+\delta}\left|\nabla \phi_{1}\right|^{p} w^{-\delta} \\
& +\beta^{p-1} \lambda_{1}\left[\left(\left(\Theta_{\alpha}^{\prime}\right)^{p-1}\right) \circ \phi_{1}\right] \cdot \phi_{1}^{p-1}\left(\beta \cdot \Theta_{\alpha} \circ \phi_{1}\right)^{\delta} w^{-\delta} \\
= & \beta^{p-1+\delta}\left\{\left|\nabla \phi_{1}\right|^{p}+\lambda_{1}\left[\left(\left(\Theta_{\alpha}^{\prime}\right)^{p-1}\right) \circ \phi_{1}\right] \cdot \phi_{1}^{p-1}\left(\Theta_{\alpha} \circ \phi_{1}\right)^{\delta}\right\} w^{-\delta} .
\end{aligned}
$$

Recall $R_{\alpha}>M=\max _{\bar{\Omega}} \phi_{1}$. The function $\Theta_{\alpha}$ being strictly increasing with strictly decreasing derivative $\Theta_{\alpha}^{\prime}$ on the interval $\left[0, R_{\alpha}\right]$, and $\Theta_{\alpha}(0)=0, \Theta_{\alpha}^{\prime}(0)=$ $\alpha>\Theta_{\alpha}^{\prime}\left(R_{\alpha}\right)=0$, we can estimate

$$
\begin{gathered}
\left(\left(\Theta_{\alpha}^{\prime}\right)^{p-1}\right) \circ \phi_{1} \geq \Theta_{\alpha}^{\prime}(M)^{p-1}>0, \\
\Theta_{\alpha} \circ \phi_{1} \geq \Theta_{\alpha}^{\prime}(M) \phi_{1} .
\end{gathered}
$$

We combine these inequalities to estimate the second summand in the curly brackets at the end of equation (A.16) above, thus obtaining

$$
-\Delta_{p} w \geq \beta^{p-1+\delta}\left\{\left|\nabla \phi_{1}\right|^{p}+\lambda_{1}\left(\Theta_{\alpha}^{\prime}(M) \phi_{1}\right)^{p-1+\delta}\right\} w^{-\delta}
$$


Moreover, we have $w \in C^{1}(\bar{\Omega})$ together with $w=0$ on $\partial \Omega, w>0$ in $\Omega$, and $\frac{\partial w}{\partial v}<0$ on $\partial \Omega$. These claims follow from $\phi_{1} \in C^{1}(\bar{\Omega})$ combined with the strong maximum and boundary point principles $\phi_{1}>0$ in $\Omega$ and $\frac{\partial \phi_{1}}{\partial v}<0$ on $\partial \Omega$ (see Vázquez [43, Theorem 5, page 200]). The same arguments render

$$
\gamma \stackrel{\text { def }}{=} \min _{\bar{\Omega}}\left\{\left|\nabla \phi_{1}\right|^{p}+\lambda_{1}\left(\Theta_{\alpha}^{\prime}(M) \phi_{1}\right)^{p-1+\delta}\right\}>0 .
$$

We choose the number $\beta>0$ large enough, such that $\beta^{p-1+\delta} \gamma \geq \lambda$. In particular, inequality (A.17) yields

$$
-\Delta_{p} w \geq \lambda w^{-\delta} \quad \text { in } \Omega .
$$

Finally, we apply the weak comparison principle to problem (3.1) (with $\underline{u}_{\lambda}$ in place of $u$ ) and to inequality (A.18) (with $w$ satisfying $w=0$ on $\partial \Omega$ ), thus arriving at $w \geq \underline{u}_{\lambda}$ a.e. in $\Omega$. We have thus verified

$$
v \leq \underline{u}_{\lambda} \leq w=\beta \cdot \Theta_{\alpha} \circ \phi_{1} \leq \alpha \beta \phi_{1} \leq c_{\lambda}^{\prime} d \quad \text { a.e. in } \Omega,
$$

where $c_{\lambda}^{\prime} \in(0, \infty)$ is a constant, as desired.

The proof of Lemma A.7 is now complete.

\section{B. Appendix}

Regularity of weak solutions to various types of degenerate elliptic partial differential equations is a broadly developed subject, with a number of general methods and results. However, when a method is applied to a particular equation, this often needs to be done in a way specific to this equation. In this appendix we consider the following quasilinear elliptic boundary value problem,

$$
-\nabla \cdot(\mathbf{a}(x, \nabla u))=f(x) \text { in } \Omega ; \quad u=0 \text { on } \partial \Omega,
$$

in a setting that is closely related to Lieberman's in [33, Theorem 1, page 1203]. We assume that $\Omega$ is a (nonempty) bounded domain in $\mathbb{R}^{N}$ whose boundary $\partial \Omega$ is a compact $C^{2}$ manifold. We denote by $x=\left(x_{1}, \ldots, x_{N}\right)$ a generic point in $\Omega$ and by $u$ the unknown function of $x$, where $u \in W_{0}^{1, p}(\Omega)$ for $p \in(1, \infty)$. The quasilinear elliptic operator $(x, u) \mapsto \nabla \cdot(\mathbf{a}(x, \nabla u))$ is defined by

$$
\nabla \cdot(\mathbf{a}(x, \nabla u)) \stackrel{\text { def }}{=} \sum_{i=1}^{N} \frac{\partial}{\partial x_{i}} a_{i}(x, \nabla u(x)) \quad \text { for } x \in \Omega \text { and } u \in W_{0}^{1, p}(\Omega)
$$

with values in $W^{-1, p^{\prime}}(\Omega)$, the dual space of $W_{0}^{1, p}(\Omega)$, where $\frac{1}{p}+\frac{1}{p^{\prime}}=1$. The components $a_{i}$ of the vector field $\mathbf{a}: \Omega \times \mathbb{R}^{N} \rightarrow \mathbb{R}^{N}, \mathbf{a}=\left(a_{1}, \ldots, a_{N}\right)$, are functions of $x$ and $\eta=\nabla u \in \mathbb{R}^{N}$, such that $a_{i} \in C^{0}\left(\Omega \times \mathbb{R}^{N}\right)$ and $\partial a_{i} / \partial \eta_{j} \in$ $C^{0}\left(\Omega \times\left(\mathbb{R}^{N} \backslash\{0\}\right)\right)$. We assume that a satisfies the following ellipticity and growth conditions: 
(H1) There exist some constants $\kappa \in[0,1], \gamma, \Gamma \in(0, \infty)$, and $\alpha \in(0,1)$, such that

$$
\begin{aligned}
a_{i}(x, 0) & =0 ; \quad i=1, \ldots, N \\
\sum_{i, j=1}^{N} \frac{\partial a_{i}}{\partial \eta_{j}}(x, \eta) \cdot \xi_{i} \xi_{j} & \geq \gamma \cdot(\kappa+|\eta|)^{p-2} \cdot|\xi|^{2}, \\
\sum_{i, j=1}^{N}\left|\frac{\partial a_{i}}{\partial \eta_{j}}(x, \eta)\right| & \leq \Gamma \cdot(\kappa+|\eta|)^{p-2}, \\
\sum_{i=1}^{N}\left|a_{i}(x, \eta)-a_{i}(y, \eta)\right| & \leq \Gamma \cdot(1+|\eta|)^{p} \cdot|x-y|^{\alpha},
\end{aligned}
$$

for all $x, y \in \Omega$, all $\eta \in \mathbb{R}^{N} \backslash\{0\}$, and all $\xi \in \mathbb{R}^{N}$.

We remark that conditions (B.3) through (B.6) are motivated by the elliptic boundary value problem

$$
-\Delta_{p} u=f(x) \text { in } \Omega ; \quad u=0 \text { on } \partial \Omega,
$$

with the $p$-Laplacian defined by $\Delta_{p} u \stackrel{\text { def }}{=} \nabla \cdot\left(|\nabla u|^{p-2} \nabla u\right)$.

Finally, we impose the following growth condition on the function $f \in L_{\mathrm{loc}}^{\infty}(\Omega)$ :

(H2) There exist constants $c$ and $\delta, 0<c<\infty$ and $0<\delta<1$, such that

$$
0 \leq f(x) \leq c d(x)^{-\delta} \quad \text { holds for almost all } x \in \Omega .
$$

It is readily seen that

$$
|d(x)-d(y)| \leq|x-y| \quad \text { for } x, y \in \bar{\Omega} .
$$

Since the boundary $\partial \Omega$ is of class $C^{2}, d$ is a $C^{2}$ function in a neighborhood of $\partial \Omega$. More precisely, we have $d \in C^{2}\left(\Gamma_{\mu}\right)$ where $\Gamma_{\mu} \stackrel{\text { def }}{=}\{x \in \bar{\Omega}: d(x)<\mu\}$, for some $\mu>0$, by Gilbarg and Trudinger [28, Lemma 14.16, page 355].

We will show the following analogue of a well-known regularity result for problem (B.1) due to Lieberman [33, Theorem 1, page 1203] (regularity near the boundary). Interior regularity was established earlier independently by DiBenedetto [19, Theorem 2, page 829] and Tolksdorf [42, Theorem 1, page 127].

Theorem B.1. Assume that $\mathbf{a}(x, \eta)$ satisfies the structural hypotheses (B.3) through (B.6), and $f(x)$ satisfies the growth hypothesis (B.8). Let $u \in W_{0}^{1, p}(\Omega)$ be the (unique) weak solution of problem (B.1). In addition, assume

$$
0 \leq u(x) \leq C d(x) \quad \text { for almost all } x \in \Omega,
$$


where $C$ is a constant, $0 \leq C<\infty$. Then there exist constants $\beta$ and $M, 0<\beta<$ $\alpha$ and $0 \leq M<\infty$, depending solely on $\Omega, N$, p, on the constants $\gamma, \Gamma, \alpha$ in (B.4) through (B.6), on the constants $c, \delta$ in (B.8), and on the constant $C$ in (B.9), but not on $\kappa \in[0,1]$, such that $u$ satisfies $u \in C^{1, \beta}(\bar{\Omega})$ and

$$
\|u\|_{C^{1, \beta}(\bar{\Omega})} \leq M
$$

It is well-known that the existence and uniqueness of a weak solution in $W_{0}^{1, p}(\Omega)$ are guaranteed by the Minty-Browder theorem for (nonlinear) monotone operators; see e.g. Deimling [16, Theorem 12.1, page 117].

We need to modify the proof of Theorem 1 from Lieberman's work [33]. In what follows we employ Lemma 5, page 1211, from [33] as it stands there, but adapt the remaining part of the proof of Theorem 1, pages 1212-1213, to our setting, in particular, equations (3.5) through (3.8).

Proof of Theorem B.1. We "flatten" the boundary $\partial \Omega$ locally by a $C^{2}$ diffeomorphism $\Phi$. Such a local transformation of coordinates, $\tilde{x}=\Phi(x)$, leaves all structural conditions for $a_{i}$ unchanged. The same remark is valid also for $f$ and $u$ in inequalities (B.8) and (B.9). In particular, we can adjust this transformation (by rotation and translation of coordinate axes) in order to achieve $d(\tilde{x})=\tilde{x}_{N}$ for all $\tilde{x} \in \mathbb{R}^{N}$ from an open ball centered at the origin and such that $\tilde{x}_{N} \geq 0$. Therefore, writing

$$
x=\left(x_{1}, \ldots, x_{N}\right) \in \mathbb{R}^{N}, x^{\prime}=\left(x_{1}, \ldots, x_{N-1}\right) \in \mathbb{R}^{N-1}, \text { and } x=\left(x^{\prime}, x_{N}\right),
$$

let us consider only an open ball

$$
B_{r}(y) \stackrel{\text { def }}{=}\left\{x \in \mathbb{R}^{N}:|x-y|<r\right\} \quad \text { for some } y \in \mathbb{R}^{N} \text { and } 0<r<\infty
$$

and the corresponding open half-ball

$$
B_{r}^{+}(y) \stackrel{\text { def }}{=}\left\{x \in B_{r}(y): x_{N}-y_{N}>0\right\}
$$

with the flat boundary portion

$$
B_{r}^{0}(y) \stackrel{\text { def }}{=}\left\{x \in B_{r}(y): x_{N}-y_{N}=0\right\} .
$$

Finally, let us introduce the half-sphere

$$
\begin{aligned}
S_{r}^{+}(y) & \stackrel{\text { def }}{=} \partial B_{r}^{+}(y) \backslash B_{r}^{0}(y) \\
& =\left\{x \in \mathbb{R}^{N}:|x-y|=r \text { and } x_{N}-y_{N} \geq 0\right\} .
\end{aligned}
$$

This means that we have replaced a general domain $\Omega$ by an open half-ball; we fix and normalize this half-ball to be $B_{1}^{+}(0) \subset \mathbb{R}^{N}$. 
We recall that the vector field

$$
\mathbf{a}:(x, \nabla u) \mapsto \mathbf{a}(x, \nabla u): B_{1}^{+}(0) \times \mathbb{R}^{N} \rightarrow \mathbb{R}^{N}
$$

satisfies the structural hypotheses (B.3) through (B.6), and the function $f: B_{1}^{+}(0)$ $\rightarrow \mathbb{R}^{N}$ verifies (B.8), i.e.,

$$
0 \leq f(x) \leq c x_{N}^{-\delta} \quad \text { holds for almost all } x=\left(x^{\prime}, x_{N}\right) \in B_{1}^{+}(0) .
$$

Hypothesis (B.9) for $u$ reads

$$
0 \leq u(x) \leq C x_{N} \quad \text { for almost all } x \in B_{1}^{+}(0) .
$$

Following Lieberman's proof of $C^{1, \beta}$ regularity near the boundary in [33], we will prove that the (unique) weak solution $u \in W^{1, p}\left(B_{1}^{+}(0)\right)$ of the partial differential equation

$$
-\nabla \cdot \mathbf{a}(x, \nabla u)=f(x) \quad \text { in } B_{1}^{+}(0) ; \quad u=0 \quad \text { on } B_{1}^{0}(0),
$$

which is assumed to obey (B.12), satisfies $\left.u \in C^{1, \beta} \overline{\left(B_{1 / 2}^{+}(0)\right.}\right)$ for some $\beta \in(0, \alpha)$. We do not specify the boundary data of $u$ on the half-sphere $S_{1}^{+}(0)$, but assume $u \in W^{1, p}\left(B_{1}^{+}(0)\right)$ instead. The method of proof is based on a standard perturbation argument using the Dirichlet boundary value problem

$$
\left\{\begin{array}{l}
-\nabla \cdot \mathbf{a}(x, \nabla v)=0 \quad \text { in } B_{R}^{+}(y) \\
v=0 \quad \text { on } B_{R}^{0}(y), \quad v=u \quad \text { on } S_{R}^{+}(y),
\end{array}\right.
$$

for any $y \in B_{1 / 2}^{+}(0)$ and any $0<R<1 / 2$; notice that $B_{R}^{+}(y) \subset B_{1}^{+}(0)$. This problem possesses a unique weak solution $v \equiv v_{R}$ in $W^{1, p}\left(B_{R}^{+}(y)\right)$. We will estimate an expression for a Campanato norm of the difference $u-v$ in $B_{R}^{+}(y)$ depending on the radius $R(0<R<1 / 2)$. Using the equivalence of Campanato and Hölder norms, we will thus be able to conclude that $u$ is in $C^{1, \beta}\left(\overline{B_{1 / 2}^{+}(0)}\right)$ for some $\beta \in(0, \alpha)$.

In order to establish the desired estimate for the Campanato expression for $u-v$ in $B_{R}^{+}(y)$, it suffices to consider the "normalized" case $y=0 \in \mathbb{R}^{N}$ and $0<R<1$. In other words, the Dirichlet boundary value problem (B.14) becomes

$$
\left\{\begin{array}{lc}
-\nabla \cdot \mathbf{a}(x, \nabla v)=0 & \text { in } B_{R}^{+}(0) \\
v=0 \quad \text { on } B_{R}^{0}(0), & v=u \quad \text { on } S_{R}^{+}(0),
\end{array}\right.
$$

with a unique weak solution $v \in W^{1, p}\left(B_{R}^{+}(0)\right)$, for any $0<R<1$.

First of all, we have $0 \leq v \leq u$ in $B_{R}^{0}(0)$, by the weak comparison principle. Hypothesis (B.12) on $u$ thus forces

$$
0 \leq u(x)-v(x) \leq C x_{N} \quad \text { for all } x=\left(x^{\prime}, x_{N}\right) \in B_{R}^{+}(0) .
$$


Subtracting equation (B.15) from (B.13), multiplying the difference by $u-v$, and finally integrating over $B_{R}^{+}(0)$, we arrive at

$$
\begin{aligned}
& \int_{B_{R}^{+}(0)}[\mathbf{a}(x, \nabla u)-\mathbf{a}(x, \nabla v)] \cdot \nabla(u-v) \mathrm{d} x \\
& =\int_{B_{R}^{+}(0)} f(x)(u-v) \mathrm{d} x \leq c C \int_{B_{R}^{+}(0)} x_{N}^{1-\delta} \mathrm{d} x=c_{1} R^{N+1-\delta},
\end{aligned}
$$

for any $0<R<1$, by (B.11) and (B.16). The constant $c_{1}=c C c_{0} \geq 0$ has been obtained using

$$
\begin{aligned}
& \int_{B_{R}^{+}(0)} x_{N}^{1-\delta} \mathrm{d} x=R^{N+1-\delta} \int_{B_{1}^{+}(0)} z_{N}^{1-\delta} \mathrm{d} z \\
& =R^{N+1-\delta} \omega_{N-1} \int_{0}^{1}\left(1-z_{N}^{2}\right)^{(N-1) / 2} z_{N}^{1-\delta} \mathrm{d} z_{N} \equiv c_{0} R^{N+1-\delta} .
\end{aligned}
$$

We estimate the left-hand side of inequality (B.17) from below as follows, applying ellipticity condition (B.4). For almost every $x \in B_{R}^{+}(0)$ we have

$$
\begin{aligned}
& {[\mathbf{a}(x, \nabla u)-\mathbf{a}(x, \nabla v)] \cdot \nabla(u-v)} \\
& =\left[\left(\int_{0}^{1} \nabla \mathbf{a}(x, \nabla(v+\theta(u-v))) \mathrm{d} \theta\right) \nabla(u-v)\right] \cdot \nabla(u-v) \\
& \geq \gamma\left(\int_{0}^{1}|\nabla(v+\theta(u-v))|^{p-2} \mathrm{~d} \theta\right)|\nabla(u-v)|^{2} .
\end{aligned}
$$

If $2 \leq p<\infty$, we obtain immediately

$$
[\mathbf{a}(x, \nabla u)-\mathbf{a}(x, \nabla v)] \cdot \nabla(u-v) \geq \gamma \kappa_{p}|\nabla(u-v)|^{p}
$$

where $\kappa_{p}>0$ is the constant from the inequality

$$
\begin{gathered}
\kappa_{p}|\mathbf{w}|^{p-2} \leq \int_{0}^{1}|\mathbf{v}+\theta \mathbf{w}|^{p-2} \mathrm{~d} \theta \quad \text { for all } \mathbf{v}, \mathbf{w} \in \mathbb{R}^{N}, \\
\kappa_{p} \stackrel{\text { def }}{=} \min _{\substack{\mathbf{v}, \mathbf{w} \in \mathbb{R}^{N} \\
|\mathbf{w}|=1}} \int_{0}^{1}|\mathbf{v}+\theta \mathbf{w}|^{p-2} \mathrm{~d} \theta>0 .
\end{gathered}
$$

We combine (B.17), (B.18), and (B.19) to get

$$
\int_{B_{R}^{+}(0)}|\nabla(u-v)|^{p} \mathrm{~d} x \leq c_{2} R^{N+1-\delta}
$$

with the constant $c_{2}=\left(\gamma \kappa_{p}\right)^{-1} c_{1} \geq 0$ independent from $0<R<1$. 
If $1<p<2$, we use the (trivial) inequality

$$
(|\mathbf{v}|+|\mathbf{w}|)^{p-2} \leq \int_{0}^{1}|\mathbf{v}+\theta \mathbf{w}|^{p-2} \mathrm{~d} \theta \quad \text { for all } \mathbf{v}, \mathbf{w} \in \mathbb{R}^{N}
$$

to obtain

$$
[\mathbf{a}(x, \nabla u)-\mathbf{a}(x, \nabla v)] \cdot \nabla(u-v) \geq \gamma(|\nabla v|+|\nabla(u-v)|)^{p-2}|\nabla(u-v)|^{2} .
$$

Next, by Hölder's inequality, we have

$$
\begin{aligned}
& \int_{B_{R}^{+}(0)}|\nabla(u-v)|^{p} \mathrm{~d} x \\
& \leq\left(\int_{B_{R}^{+}(0)}(|\nabla v|+|\nabla(u-v)|)^{p-2}|\nabla(u-v)|^{2} \mathrm{~d} x\right)^{p / 2} \\
& \quad \times\left(\int_{B_{R}^{+}(0)}(|\nabla v|+|\nabla(u-v)|)^{p} \mathrm{~d} x\right)^{(2-p) / 2},
\end{aligned}
$$

and then, applying (B.21) followed by Minkowski's inequality,

$$
\begin{aligned}
& \int_{B_{R}^{+}(0)}|\nabla(u-v)|^{p} \mathrm{~d} x \\
& \leq \gamma^{-p / 2}\left(\int_{B_{R}^{+}(0)}[\mathbf{a}(x, \nabla u)-\mathbf{a}(x, \nabla v)] \cdot \nabla(u-v) \mathrm{d} x\right)^{p / 2} \\
& \quad \times\left(\int_{B_{R}^{+}(0)}(|\nabla v|+|\nabla(u-v)|)^{p} \mathrm{~d} x\right)^{(2-p) / 2} \\
& \leq\left(\frac{c_{1} R^{N+1-\delta}}{\gamma}\right)^{p / 2}\left(\int_{B_{R}^{+}(0)}(|\nabla v|+|\nabla(u-v)|)^{p} \mathrm{~d} x\right)^{(2-p) / 2} \\
& \leq\left(\frac{c_{1} R^{N+1-\delta}}{\gamma}\right)^{p / 2}\left[\left(\int_{B_{R}^{+}(0)}|\nabla v|^{p} \mathrm{~d} x\right)^{1 / p}+\left(\int_{B_{R}^{+}(0)}|\nabla(u-v)|^{p} \mathrm{~d} x\right)^{1 / p}\right]^{(2-p) p / 2} .
\end{aligned}
$$

With the notation

$$
J(u-v ; R)=\int_{B_{R}^{+}(0)}|\nabla(u-v)|^{p} \mathrm{~d} x \quad \text { and } \quad J(v ; R)=\int_{B_{R}^{+}(0)}|\nabla v|^{p} \mathrm{~d} x,
$$

this inequality simplifies to

$$
\begin{aligned}
J(u-v ; R)^{\frac{2}{(2-p) p}} & =J(u-v ; R)^{\frac{1}{p}+\frac{1}{2-p}} \\
& \leq\left(c_{3} R^{N+1-\delta}\right)^{1 /(2-p)}\left(J(u-v ; R)^{1 / p}+J(v ; R)^{1 / p}\right)
\end{aligned}
$$


whenever $0<R<1$, where we have introduced $c_{3}=\gamma^{-1} c_{1} \geq 0$. Substituting

$$
J(u-v ; R)=c_{3} R^{N+1-\delta} \tilde{J}(u-v ; R) \quad \text { and } \quad J(v ; R)=c_{3} R^{N+1-\delta} \tilde{J}(v ; R)
$$

in the last inequality, we obtain

$$
\tilde{J}(u-v ; R)^{\frac{1}{p}+\frac{1}{2-p}} \leq \tilde{J}(u-v ; R)^{1 / p}+\tilde{J}(v ; R)^{1 / p}
$$

whenever $0<R<1$. Examining the alternatives

$$
\tilde{J}(u-v ; R) \geq \tilde{J}(v ; R)^{1 / p} \quad \text { and } \quad \tilde{J}(u-v ; R) \leq \tilde{J}(v ; R)^{1 / p},
$$

from this inequality we deduce further

$$
\tilde{J}(u-v ; R) \leq \max \left\{2^{2-p}, 2^{(2-p) p / 2} \tilde{J}(v ; R)^{(2-p) / 2}\right\},
$$

whenever $0<R<1$, and consequently

$$
\begin{aligned}
& J(u-v ; R) \\
& \leq c_{3} R^{N+1-\delta} \cdot \max \left\{2^{2-p}, 2^{(2-p) p / 2}\left(c_{3} R^{N+1-\delta}\right)^{-(2-p) / 2} J(v ; R)^{(2-p) / 2}\right\} \\
& =c_{3} R^{N+\frac{(1-\delta) p}{2}} \cdot \max \left\{2^{2-p} R^{\frac{(1-\delta)(2-p)}{2}}, 2^{(2-p) p / 2}\left(c_{3}^{-1} R^{-N} J(v ; R)\right)^{(2-p) / 2}\right\}
\end{aligned}
$$

which yields

$$
R^{-N} J(u-v ; R) \leq c_{4} R^{(1-\delta) p / 2} \cdot \max \left\{1,\left(R^{-N} J(v ; R)\right)^{(2-p) / 2}\right\}
$$

where $c_{4} \geq 0$ is a constant independent from $0<R<1$.

Applying certain estimates on suitable norms of $v$ from [33, Lemma 5, page 1211], Lieberman has derived the following inequality for $J(v ; \cdot):(0,1) \rightarrow[0, \infty)$, see [33, Inequality (3.6), page 1212]:

$$
J(v ; r) \leq C_{0}\left\{R^{N}+(r / R)^{N} J(v ; R)\right\} \quad \text { for all } 0<r<R \leq R_{0},
$$

where $C_{0} \geq 0$ and $0<R_{0}<1$ are constants independent from both $r$ and $R$. By Lemma B.2 below, this implies

$$
\sup _{0<R \leq R_{0}} R^{-N+\eta} J(v ; R)=\sup _{0<R \leq R_{0}} \frac{1}{R^{N-\eta}} \int_{B_{R}^{+}(0)}|\nabla v|^{p} \mathrm{~d} x \equiv C(\eta)<\infty
$$

for any number $0<\eta<N$. Finally, we apply this inequality to (B.22), thus arriving at

$$
J(u-v ; R) \leq c_{5} R^{N+\mu(1-\delta)}
$$


where $c_{5} \equiv c_{5}\left(R_{0}\right) \geq 0$ is a constant independent from $0<R \leq R_{0}, \mu=$ $\frac{1}{2}\left(p-\frac{(2-p) \eta}{1-\delta}\right)$ satisfies $0<\mu<p / 2$, and $\eta$ needs to be taken such that $0<\eta<$ $\frac{p}{2-p}(1-\delta)$ in order to guarantee $\mu>0$.

Inequality (B.24) holds for any $1<p<\infty$. We summarize inequalities (B.20) and (B.25) to obtain, for any $1<p<\infty$,

$$
J(u-v ; R) \leq c_{5} R^{N+\mu(1-\delta)}
$$

where $\mu=1$ if $2 \leq p<\infty, 0<\mu<p / 2$ if $1<p<2$, and $c_{5} \equiv c_{5}\left(R_{0}\right) \geq 0$ is a constant independent from $0<R \leq R_{0}$.

The proof of regularity of $u$, i.e., $u \in C^{1, \beta}\left(\overline{B_{1 / 2}^{+}(0)}\right)$, can now be completed exactly as in the work of Lieberman [33, page 1213] or DiBenedetto [19, page 849], again with a help from certain estimates on suitable norms of $v$ obtained in [33, Lemma 5, page 1211].

It remains to prove $(\mathrm{B} .23) \Longrightarrow(\mathrm{B} .24)$. This is an easy consequence of the following lemma. We denote $\mathbb{R}_{+}=[0, \infty)$.

Lemma B.2. Let $J:[0,1] \rightarrow \mathbb{R}_{+}$be a function which satisfies the following inequality

$$
J(r) \leq C\left\{R^{N}+\left[\theta(R)+(r / R)^{N}\right] J(R)\right\}
$$

for all $0 \leq r<R \leq 1$, where $C \geq 0$ and $N>0$ are some constants, and $\theta:[0,1] \rightarrow \mathbb{R}_{+}$is a monotone decreasing function with $\theta(R) \searrow 0$ as $R \searrow 0$. Then, for any $0<\eta<N$, there exists a constant $C(\eta) \geq 0$ such that

$$
J(R) \leq C(\eta) R^{N-\eta} \quad \text { for all } 0<R \leq 1 .
$$

Proof. Fix any number $\eta$ with $0<\eta<N$. We choose $0<R_{0} \leq 1$ and $0<t_{0} \leq 1$ such that

$$
C\left[\theta\left(R_{0}\right)+t_{0}^{N}\right] \leq \frac{1}{2} t_{0}^{N-\eta}
$$

Hence, by our hypothesis on the function $\theta$, we have

$$
C\left[\theta(R)+t^{N}\right] \leq \frac{1}{2} t^{N-\eta}
$$

for all $t$ and $R$ with $0<t \leq t_{0}$ and $0<R \leq R_{0}$. We infer from inequality (B.27) that

$$
J(t R) \leq C R^{N}+\frac{1}{2} t^{N-\eta} J(R)
$$

and therefore also

$$
\frac{J(t R)}{(t R)^{N-\eta}} \leq \frac{C R^{\eta}}{t^{N-\eta}}+\frac{1}{2} \frac{J(R)}{R^{N-\eta}}
$$


whenever $0<t \leq t_{0}$ and $0<R \leq R_{0}$. Replacing $R$ by $t^{j} R$ for $j=0,1, \ldots, k-1$ in this estimate, we obtain by induction on $k=1,2, \ldots$ that

$$
\frac{J\left(t^{k} R\right)}{\left(t^{k} R\right)^{N-\eta}} \leq \frac{C R^{\eta}}{t^{N-\eta}} \sum_{j=0}^{k-1} 2^{-j}+\frac{1}{2^{k}} \frac{J(R)}{R^{N-\eta}} \leq \frac{2 C R^{\eta}}{t^{N-\eta}}+\frac{J(R)}{2 R^{N-\eta}}
$$

whenever $0<t \leq t_{0}$ and $0<R \leq R_{0}$. Fixing $t=t_{0}$ and $R=R_{0}$ we arrive at

$$
\frac{J\left(t_{0}^{k} R_{0}\right)}{\left(t_{0}^{k} R_{0}\right)^{N-\eta}} \leq C_{0}(\eta) \stackrel{\text { def }}{=} \frac{2 C R_{0}^{\eta}}{t_{0}^{N-\eta}}+\frac{1}{R_{0}^{N-\eta}} \cdot \sup _{0<R \leq R_{0}} J(R)<\infty
$$

for every $k=0,1,2, \ldots$

Finally, if $t_{0}^{k} R_{0} \leq r<t_{0}^{k-1} R_{0}$ for some $k \geq 1$, we first apply inequality (B.27) with $t_{0}^{k-1} R_{0}$ in place of $R$ to get

$$
J(r) \leq C\left\{\left(t_{0}^{k-1} R_{0}\right)^{N}+\left[\theta\left(t_{0}^{k-1} R_{0}\right)+\left(\frac{r}{t_{0}^{k-1} R_{0}}\right)^{N}\right] J\left(t_{0}^{k-1} R_{0}\right)\right\}
$$

and then combine the result with inequality (B.30) to obtain

$$
\begin{aligned}
J(r) & \leq C\left\{\left(t_{0}^{k-1} R_{0}\right)^{N}+\left[\theta\left(t_{0}^{k-1} R_{0}\right)+1\right] C(\eta)\left(t_{0}^{k-1} R_{0}\right)^{N-\eta}\right\} \\
& \leq C\left\{\left(r / t_{0}\right)^{N}+\left[\theta\left(R_{0}\right)+1\right] C(\eta)\left(r / t_{0}\right)^{N-\eta}\right\} \\
& =C\left(r / t_{0}\right)^{N-\eta}\left\{\left(r / t_{0}\right)^{\eta}+C(\eta)\left[\theta\left(R_{0}\right)+1\right]\right\} \\
& \leq C\left\{\left(R_{0} / t_{0}\right)^{\eta}+C(\eta)\left[\theta\left(R_{0}\right)+1\right]\right\}\left(r / t_{0}\right)^{N-\eta} .
\end{aligned}
$$

The desired estimate (B.28) follows immediately.

\section{Appendix}

The following result is a standard argument which gives sufficient conditions guaranteeing that a weakly convergent Palais-Smale sequence in $W_{0}^{1, p}(\Omega)$ is also strongly convergent.

Lemma C.1. Let $1<p<\infty$ and let $F: W_{0}^{1, p}(\Omega) \rightarrow W^{-1, p^{\prime}}(\Omega)$ be a mapping, such that both functionals $u \mapsto\langle F(u), u\rangle$ and $u \mapsto\|F(u)\|_{W^{-1, p^{\prime}(\Omega)}}$ are 
weakly lower semicontinuous on $W_{0}^{1, p}(\Omega)$. Define the mapping $T: W_{0}^{1, p}(\Omega) \rightarrow$ $W^{-1, p^{\prime}}(\Omega)$ :

$$
T(u) \stackrel{\text { def }}{=}-\Delta_{p} u+F(u), \quad u \in W_{0}^{1, p}(\Omega) .
$$

Finally, assume that $\left\{u_{k}\right\}_{k=1}^{\infty} \subset W_{0}^{1, p}(\Omega)$ is a sequence satisfying, as $k \rightarrow \infty$,

(i) $\left\|T\left(u_{k}\right)\right\|_{W^{-1, p^{\prime}(\Omega)}} \rightarrow 0$;

(ii) $\left\|u_{k}\right\|_{W_{0}^{1, p}(\Omega)} \rightarrow \ell>0$.

Then $\left\{u_{k}\right\}_{k=1}^{\infty}$ possesses a strongly convergent subsequence.

Proof. Owing to (ii), $\left\{u_{k}\right\}_{k=1}^{\infty}$ possesses a weakly convergent subsequence, denoted again by $\left\{u_{k}\right\}_{k=1}^{\infty}, u_{k} \rightarrow u_{0}$ weakly in $W_{0}^{1, p}(\Omega)$ as $k \rightarrow \infty$. Next, we employ the identity

$$
\begin{aligned}
\langle T(u)-F(u), u\rangle & =\left\langle-\Delta_{p} u, u\right\rangle=\int_{\Omega}|\nabla u|^{p} \mathrm{~d} x \\
& =\left\|-\Delta_{p} u\right\|_{W^{-1, p^{\prime}}(\Omega)}\|u\|_{W_{0}^{1, p}(\Omega)} \\
& =\|T(u)-F(u)\|_{W^{-1, p^{\prime}}(\Omega)}\|u\|_{W_{0}^{1, p}(\Omega)}
\end{aligned}
$$

for $u \in W_{0}^{1, p}(\Omega)$. We take $u=u_{k}$ and let $k \rightarrow \infty$, thus obtaining

$$
\begin{aligned}
-\left\langle F\left(u_{0}\right), u_{0}\right\rangle & \geq-\limsup _{k \rightarrow \infty}\left\langle F\left(u_{k}\right), u_{k}\right\rangle=\liminf _{k \rightarrow \infty}\left\langle-F\left(u_{k}\right), u_{k}\right\rangle \\
& =\liminf _{k \rightarrow \infty}\left\langle T\left(u_{k}\right)-F\left(u_{k}\right), u_{k}\right\rangle=\liminf _{k \rightarrow \infty} \int_{\Omega}\left|\nabla u_{k}\right|^{p} \mathrm{~d} x \\
& =\left(\liminf _{k \rightarrow \infty}\left\|T\left(u_{k}\right)-F\left(u_{k}\right)\right\|_{W^{-1, p^{\prime}}(\Omega)}\right)\left(\lim _{k \rightarrow \infty}\left\|u_{k}\right\|_{W_{0}^{1, p}(\Omega)}\right) \\
& =\ell \cdot \liminf _{k \rightarrow \infty}\left\|F\left(u_{k}\right)\right\|_{W^{-1, p^{\prime}}(\Omega)} \geq \ell \cdot\left\|F\left(u_{0}\right)\right\|_{W^{-1, p^{\prime}}(\Omega)},
\end{aligned}
$$

by (i). It follows that $\left\|u_{0}\right\|_{W_{0}^{1, p}(\Omega)} \geq \ell$ provided we can show $F\left(u_{0}\right) \neq 0$ in $W^{-1, p^{\prime}}(\Omega)$. Indeed, $\left\|F\left(u_{0}\right)\right\|_{W^{-1, p^{\prime}}(\Omega)}=0$ would force

$$
\liminf _{k \rightarrow \infty}\left\|u_{k}\right\|_{W_{0}^{1, p}(\Omega)}^{p}=\liminf _{k \rightarrow \infty} \int_{\Omega}\left|\nabla u_{k}\right|^{p} \mathrm{~d} x=0,
$$

thus contradicting $\ell>0$ in (ii). 
Finally, we combine $u_{k} \rightarrow u_{0}$ in $W_{0}^{1, p}(\Omega)$ with the inequalities

$$
0<\ell \leq\left\|u_{0}\right\|_{W_{0}^{1, p}(\Omega)} \leq \liminf _{k \rightarrow \infty}\left\|u_{k}\right\|_{W_{0}^{1, p}(\Omega)}=\ell
$$

to conclude that $u_{k} \rightarrow u_{0}$ strongly in $W_{0}^{1, p}(\Omega)$.

\section{References}

[1] Adimurthi and J. Giacomoni, Multiplicity of positive solutions for a singular and critical elliptic problem in $\mathbb{R}^{2}$, Commun. Contemp. Math. 8 (2006), 621-656.

[2] A. Ambrosetti, H. Brezis and G. Cerami, Combined effects of concave and convexe nonlinearities in some elliptic problems, J. Funct. Anal. 122 (1994), 519-543.

[3] A. Ambrosetti, J. P. García Azorero and I. Peral Alonso, Multiplicity results for some nonlinear elliptic equations, J. Funct. Anal. 137 (1996), 219-242.

[4] A. AmbrosetTI and P. H. RABINOWITZ, Dual variational methods in critical point theory and applications, J. Funct. Anal. 14 (1973), 349-381.

[5] A. ANANE, Simplicité et isolation de la première valeur propre du p-laplacien avec poids, C.R. Acad. Sci. Paris, Sér. I-Math. 305 (1987), 725-728.

[6] A. AnANE, "Etude des valeurs propres et de la résonance pour l'opérateur $p$-Laplacien", Thèse de doctorat, Université Libre de Bruxelles, 1988, Brussels.

[7] C. ARANDA and T. GODOY, Existence and multiplicity of positive solutions for a singular problem associated to the p-Laplacian operator, Electron. J. Differential Equations 132 (2004), 1-15.

[8] F. V. AtKinson and L. A. Peletier, Emden-Fowler equations involving critical exponents, Nonlinear Anal. 10 (1986), 755-776.

[9] L. BOCCARDO and F. MURAT, Almost everywhere convergence of the gradients of solutions to elliptic and parabolic equations, Nonlinear Anal. 19 (1992), 581-597.

[10] $\mathrm{H}$. BREZIS and E. LIEB, A relation between point convergence of functions and convergence of functionals, Proc. Amer. Math. Soc. 88 (1983), 486-490.

[11] H. Brezis and L. Nirenberg, Positive solutions of nonlinear elliptic equation involving the critical Sobolev exponent, Comm. Pure Appl. Math. 36 (1983), 437-477.

[12] H. Brezis and L. Nirenberg, Minima locaux relatifs à $C^{1}$ et $H^{1}$, C.R. Acad. Sci. Paris, Sér. I-Math. 317 (1993), 465-472.

[13] M. M. Coclite and G. PAlmieri, On a singular nonlinear Dirichlet problem, Comm. Partial Differential Equations 14 (1989), 1315-1327.

[14] M. CUESTA and P. TAKÁČ, A strong comparison principle for positive solutions of degenerate elliptic equations, Differential Integral Equations 13 (2000), 721-746.

[15] M. G. Crandall, P. H. Rabinowitz and L. TARTar, On a Dirichlet problem with a singular nonlinearity, Comm. Partial Differential Equations 2 (1977), 193-222.

[16] K. Deimling, "Nonlinear Functional Analysis", Springer-Verlag, Berlin-HeidelbergNew York, 1985.

[17] J. I. DÍAZ and J. E. Saá, Existence et unicité de solutions positives pour certaines équations elliptiques quasilinéaires, C.R. Acad. Sci. Paris, Sér. I-Math. 305 (1987), 521-524.

[18] J. I. DÍAZ, J. M. MOREL and L. OswALD, An elliptic equation with singular nonlinearity, Comm. Partial Differential Equations 12 (1987), 1333-1344.

[19] E. DiBenedetTo, $C^{1+\alpha}$ local regularity of weak solutions of degenerate elliptic equations, Nonlinear Anal. 7 (1983), 827-850.

[20] J. P. García Azorero and I. Peral Alonso, Some results about the existence of a second positive solution in a quasilinear critical problem, Indiana Univ. Math. J. 43 (1994), 941-957. 
[21] J. P. García Azorero, I. Peral Alonso and J. J. MAnfredi, Sobolev versus Hölder local minimizers and global multiplicity for some quasilinear elliptic equations, Commun. Contemp. Math. 2 (2000), 385-404.

[22] J. GiACOMONI and K. SREENADH, Multiplicity results for a singular and quasilinear equation, submitted for publication.

[23] M. Giaquinta, "Multiple Integrals in the Calculus of Variations and Nonlinear Elliptic Systems", Annals of Mathematics Studies, Princeton University Press, Princeton, N.J., 1983.

[24] M. Giaquinta and E. GiUsti, Global $C^{1+\alpha}$-regularity for second order quasilinear elliptic equations in divergence form, J. Reine Angew. Math. 351 (1984), 55-65.

[25] N. Ghoussoub and D. PreIss, A general mountain pass principle for locating and classifying critical points, Ann. Inst. H. Poincaré Anal. Non Linéaire 6 (1989), 321-330.

[26] N. GHoussoub and C. YuAN, Multiple solutions for quasilinear PDEs involving the critical Sobolev and Hardy Exponents, Trans. Amer. Math. Soc. 352 (2000), 5703-5743.

[27] M. GuedDa and L. VÉron, Quasilinear elliptic equations involving critical Sobolev exponents, Nonlinear Anal. 13 (1989), 879-902.

[28] D. Gilbarg and N. S. Trudinger, "Elliptic Partial Differential Equations of Second Order", Springer-Verlag, New-York, 1983.

[29] Y. HAITAO, Multiplicity and asymptotic behavior of positive solutions for a singular semilinear elliptic problem, J. Differential Equations 189 (2003), 487-512.

[30] J. Hernández, F. MAncebo and J. M. Vega, On the linearization of some singular, nonlinear elliptic problems and applications, Ann. Inst. H. Poincaré Anal. Non Linéaire 19 (2002), 777-813.

[31] N. HirANO, C. SACCON and N. SHIOJI, Existence of multiple positive solutions for singular elliptic problems with concave and convex nonlinearities, Adv. Differential Equations 9 (2004), 197-220.

[32] A. C. LAZER and P. J. MCKenNA, On a singular nonlinear elliptic boundary value problem, Proc. Amer. Math. Soc. 111 (1991), 721-730.

[33] G. Lieberman, Boundary regularity for solutions of degenerate elliptic equations, Nonlinear Anal. 12 (1988), 1203-1219.

[34] J. Moser, A sharp form of an inequality by N. Trudinger, Indiana Univ. Math. J. 20 (1971), 1077-1092.

[35] Z. NeHARI, On a class of nonlinear second order differential equations, Trans. Amer. Math. Soc. 95 (1960), 101-123.

[36] R. R. Phelps, "Convex Functions, Monotone Operators, and Differentiability", Lecture notes in Mathematics, Vol. 1364, Springer-Verlag, Berlin, 1993.

[37] S. Prashanth and K. SReEnAdH, Multiplicity Results in a ball for p-Laplace equation in a ball with positive nonlinearity, Adv. Differential Equations 7 (2002), 877-896.

[38] I. SCHINDLER, Quasilinear elliptic boundary-value problems on unbounded cylinders and a related mountain-pass lemma, Arch. Rational Mech. Anal. 120 (1992), 363-374.

[39] J. B. SERRIN, Local behavior of solutions of quasilinear elliptic equations, Acta Math. 111 (1964), 247-302.

[40] P. TAKÁČ, On the Fredholm alternative for the p-Laplacian at the first eigenvalue, Indiana Univ. Math. J. 51 (2002), 187-237.

[41] P. TOLKSDORF, On the Dirichlet problem for quasilinear equations in domans with conical boundary points, Comm. Partial Differential Equations 8 (1983), 773-817.

[42] P. TOLKSDORF, Regularity for a more general class of quasilinear elliptic equations, J. Differential Equations 51 (1984), 126-150.

[43] J. L. VÁZQUEZ, A strong maximum principle for some quasilinear elliptic equations, Appl. Math. Optim. 12 (1984), 191-202. 
[44] S. YiJing, W. ShaOpING and L. YIMING, Combined effects of singular and superlinear nonlinearities in some singular boundary value problems, J. Differential Equations 176 (2001), 511-531.

\author{
MIP-CEREMATH \\ (UMR 5640)/bat C \\ Manufacture des Tabacs \\ Allée de Brienne 21 \\ F-31000 Toulouse, France \\ jgiacomo@univ-tlse1.fr \\ Ian.Schindler@univ-tlse1.fr \\ Fachbereich Mathematik \\ Universität Rostock \\ Universitätsplatz 1 \\ D-18055 Rostock, Germany \\ peter.takac@uni-rostock.de
}

